\title{
Differential Space-Time Network Coding for Multi-Source Cooperative Communications
}

\author{
Zhenzhen Gao, Student Member, IEEE, Hung-Quoc Lai, Member, IEEE, and K. J. Ray Liu, Fellow, IEEE
}

\begin{abstract}
Due to the asynchronous nature of cooperative communications, simultaneous transmissions from two or more nodes are challenging in practice. The existing cooperative communications employing successive transmission from one user node to the other can avoid the synchronization problem but results in large transmission delay. In addition, channel estimation in multisource cooperative communications is a challenging and costly task due to the amount of training, especially when the number of cooperative users is large. Considering these practical challenges in multi-source cooperative communications, this paper proposes a differential space-time network coding (DSTNC) scheme for narrowband multi-source cooperative communications to overcome the problems of imperfect synchronization and complex channel estimation without introducing large transmission delay. Each user in the network linearly combines the correctly decoded symbols via network coding and transmits its packet in time division multiple access (TDMA) mode. The pairwise error probability is analyzed and the design criteria of the DSTNC are derived to achieve full diversity. For broadband cooperative communications, distributed differential space-timefrequency network coding (DSTFNC), which is differentially encoded within each orthogonal frequency-division multiplexing (OFDM) block, is designed through mapping from the proposed DSTNC. When the statistical channel power-delay profile is known at the corresponding user node, each node can permutate its channel independently to improve the performance of the DSTFNC scheme. Simulation results are presented to verify the performance of the proposed schemes.
\end{abstract}

Index Terms-Multinode cooperative communications, synchronization, channel estimation, space-time network coding, space-time-frequency network coding.

\section{INTRODUCTION}

I $\mathrm{T}$ is well known that due to the fading effect, the transmission over wireless channels suffers from severe attenuation in signal strength. Spatial diversity is an attractive way to combat fading in wireless communications because it can be readily combined with other forms of diversity and still offer dramatic performance gains when other forms of diversity are unavailable [1]. Multiple-input multiple-output (MIMO) with space-time coding techniques exploit spatial diversity through multiple transmit and/or receive antennas [2]-[3]. However

Paper approved by G. Bauch, the Editor for MIMO, Coding and Relaying of the IEEE Communications Society. Manuscript received November 8, 2010; revised April 28, 2011.

Z. Gao and K. J. R. Liu are with the Department of Electrical and Computer Engineering, University of Maryland, College Park, MD 20742 USA (e-mail: \{ygggzhen, kjrliu\}@umd.edu). Z. Gao is also with the School of Electronic and Information Engineering, Xi'an Jiaotong University, Xi'an, China, 710049. This work was done during her visit to the University of Maryland.

H.-Q. Lai is with the US Army RDECOM CERDEC, RDER-STADS, Bldg. 242, Aberdeen Proving Ground, MD 21005 USA (e-mail: hungquoc.lai@us.army.mil).

Digital Object Identifier 10.1109/TCOMM.2011.082111.100694 with the required separation among antennas that is usually at least a half of wavelength, it is often impractical to equip multiple antennas for small size transceivers such as nodes in wireless sensor networks and mobile networks.

User cooperation is an effective scheme to introduce spatial diversity in wireless networks without the use of co-located multiple antennas. The distributed antennas from the relays form a virtual antenna array to provide spatial diversity. Most of the existing studies assume perfect synchronization and perfect channel state information (CSI) available at the destination [4], [5]. However, such an assumption is difficult to be met in practice. The cooperative diversity is provided by different antennas in separated terminals, where each terminal has its own local oscillator (LO). These LO's generate transmit frequencies with certain variations, and it is hard for the destination to estimate and compensate all the frequency offsets at once. The frequency synchronization issue becomes much worse when cooperative nodes are in mobile with different Doppler effects. It is also challenging for the destination to receive all relaying signals simultaneously due to different propagation time, processing time, and timing estimation errors. Besides, channel estimation is a challenging and costly task, especially in time-selective fading environments [6]. The amount of overhead becomes substantial in multisource wireless systems because the amount of training or convergence time (incurred by blind techniques) grows with the number of links.

Recently, there have been studies on space-time coding for asynchronous cooperative systems to achieve full diversity. Coding methods that tolerate the timing synchronization errors are discussed in [7], and [8]. However, these schemes come with the additional overheads to accommodate the timing mismatches. In [9], intentional delays are introduced in different relay nodes, and minimum mean square error (MMSE) estimator is used at the destination to exploit the cooperative diversity. However, full diversity is not guaranteed. To achieve full diversity in frequency-selective channels without perfect synchronization, [10] proposed the randomized space-time codes, and [11] exploited the structure of time-reverse spacetime block code (TR-STBC). Both of the transmissions are structured in blocks of appropriate length to eliminate interblock interference. However, if space-time block codes are used in [10], the transmission rates of [10] and [11] decrease as the number of relays increases. Full-diversity multisource cooperation protocols have been proposed in [12] and [13] based on complex field coding to improve spectral efficiency. To reduce the transmission delay caused by traditional TDMA transmission, novel concepts of wireless network 


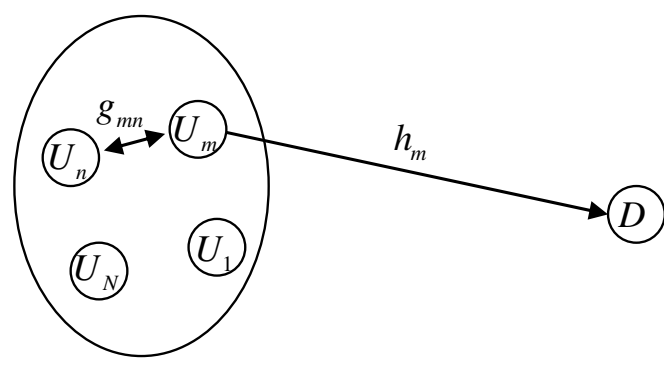

Fig. 1. Wireless multi-source cooperative communication network.

cocast (WNC) and space-time network coding (STNC) have been proposed by leveraging the idea of network coding in [14] and [15]. WNC in [14] can provide incremental diversity, while the STNC scheme in [15], which combines symbols in code division multiple access (CDMA) or frequency division multiple access (FDMA) mode at each relay, can solve the imperfect synchronization issue and achieve full diversity. $2 \mathrm{~N}$ time slots are required by the STNC in [15] for a network of $N$ nodes to transmit $N$ packets. Compared to $N^{2}$ time slots for a traditional TDMA-based cooperative communication network of $N$ nodes [5], this is a substantial reduction in transmission delay. However, in both [14] and [15], extra frequency resource is required.

All these asynchronous schemes have assumed CSI known at the receivers. However, as mentioned in previous part, channel estimation is challenging and often not possible in a fast-fading environment. To overcome the problem of channel estimation, differential encoding and modulation schemes for cooperative communications have been investigated, such as [16] and [17]. The differential scheme in [16] is based on space-time codes for MIMO systems, which would fail if the synchronization is imperfect. In [17], a single transmission with differential M-array phase shift keying (DMPSK) modulated symbols in TDMA manner can overcome the synchronization issue but causes large transmission delay. From the above discussion, there is a clear need of a cooperative communication scheme that can leverage the advantages of the discussed schemes while overcoming their shortages.

In this paper, we first propose a differential space-time network coding (DSTNC) scheme for narrowband cooperative communication systems to solve the issues of imperfect synchronization and channel estimation. By exploiting the idea of STNC in [15], TDMA is also used in this paper. The differential encoding is performed across the symbols within the same packet at each node. Different from the STNC in [15], a combining function, which is determined by a network coding vector, is designed in this paper for each node to reduce the transmission delay while achieving full diversity. Note that due to the adoption of FDMA or CDMA in [15], more bandwidth is needed by the STNC scheme to separate different users' signals. In our proposed DSTNC scheme, the same frequency band is used for the whole network and thus the proposed DSTNC scheme has higher spectral efficiency than the STNC in [15]. The network coding here, which is a set of complex parameters optimized for conventional signal constellations, is different from the traditional network coding, which linearly combines Galois symbols with coding coefficients picked from a finite field of fixed size [18], [19]. We analyze the pairwise error probability (PEP) performance of the proposed DSTNC scheme and derive the diversity criterion and product criterion for the design of the DSTNC. It is verified by the simulations that full diversity can be achieved by the proposed DSTNC scheme.

For broadband cooperative communications, orthogonal frequency-division multiplexing (OFDM) is used at each user node. A distributed differential space-time-frequency network code (DSTFNC) is designed from the proposed DSTNC via mapping. The DSTFNC is differentially encoded over subcarriers within each OFDM block. Therefore, it can be decoded even the fading channels remain constant only within each OFDM block and may change independently from one OFDM block to another. When a statistical channel powerdelay profile (PDP) is known at the corresponding user node, a smooth logical channel similar to [20] is created independently at each user node to improve the performance of the DSTFNC scheme. Simulation results show that the proposed DSTFNC scheme provides the expected full diversity even in some high mobility scenarios, where the channel may change within one OFDM block due to Doppler effects.

The rest of the paper is organized as follows. In Section II, system model and differential transmission for narrow-band cooperative communications are introduced. In Section III, the PEP performance is analyzed for frequency flat channels and the design of DSTNC is discussed. In Section IV, DSTFNC for broadband cooperative communications is generated from DSTNC and the permutation method for each node to create the smooth logical channel is illustrated when the corresponding PDP is known. In Section V, simulation results are shown to validate the proposed schemes. Finally, we draw our conclusions in Section VI.

Notations: Upper (lower) case boldface letters stand for matrices (vectors). $\otimes$ is the Kronecker product. $\mathrm{T}$ and $\mathrm{H}$ denote transposition and conjugate transposition of a matrix, respectively. E stands for expectation. $\|\mathbf{A}\|_{\mathrm{F}}$ denotes the Frobenius norm of matrix A. $\mathbf{I}_{N}$ is an $N \times N$ unit matrix. $\mathbf{0}_{N}$ and $\mathbf{1}_{N}$ are $N \times 1$ all-zero vector and all-one vector, respectively. $\operatorname{diag}\left[d_{1}, \ldots, d_{N}\right]$ is the $N \times N$ diagonal matrix whose $i$ th diagonal entry is $d_{i}$.

\section{SySTEM MODEL}

Consider a wireless multi-source cooperative communication network depicted in Fig. 1, in which a set of $N$ user nodes $U_{i}, 1 \leq i \leq N$, each equipped with a single antenna, cooperate to transmit their information to the destination node $D$. Without loss of generality, the transmitted information can be represented in terms of symbols. Nevertheless, the nodes will transmit packets that contains a large number of symbols. The destination will collect all the transmitted packets and then jointly detect the transmitted information as in the traditional network coding [18], [19]. Selective decode-and-forward protocol [1] is adopted in the cooperative communication network. For mathematical tractability, we 


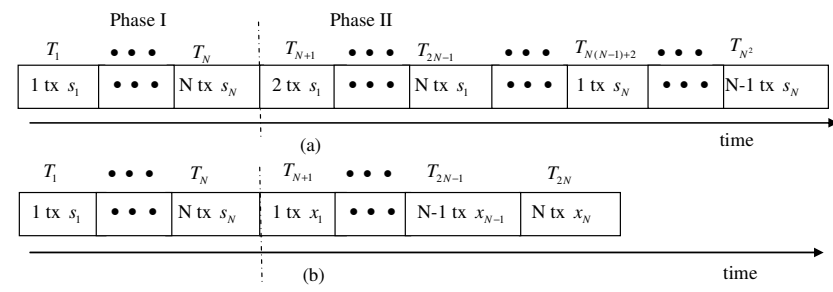

Fig. 2. Illustration of two-phase cooperative communications of repetitionbased successive transmissions (a) and network coding transmissions (b).

assume that the relays can judge whether the received symbols are decoded correctly or not [5]. It is shown in [5] that this assumption is very close to the performance of practical scenario of comparing the received SNR to a threshold, especially when the nodes operate in high SNR, for example, when the nodes are close to each other. The channels between any two nodes in the network are modeled as narrow-band Rayleigh fading with additive white Gaussian noise (AWGN). Let $g_{m n}$ and $h_{m}$ denote the channel between $U_{m}$ and $U_{n}$ and the channel between $U_{m}$ and $D, \forall m, n \in[1, N]$, respectively. Then $g_{m n}$ and $h_{m}$ are modeled as independent zero-mean, complex Gaussian random variables with variances $\mathrm{E}\left|g_{m n}\right|^{2}=\sigma_{m n}^{2}$ and $\mathrm{E}\left|h_{m}\right|^{2}=\sigma_{m}^{2}$, respectively. The channel variances are $\sigma_{m n}^{2}=\kappa d_{m n}^{-\nu}$ and $\sigma_{m}^{2}=\kappa d_{m}^{-\nu}$, where $\kappa$ is a constant whose value depends on the propagation environment, $d_{m n}$ and $d_{m}$ is the distance between $U_{m}$ and $U_{n}$ and the distance between $U_{m}$ and $D$, respectively, and $\nu$ is the path loss exponent, whose value is usually in the range between 2 and 4 .

\section{A. Transmission Model}

Fig. 2 illustrates the transmission procedure for a twophase cooperative communication. Each transmission period comprises two phases, the broadcasting phase (Phase I) and the encoding/relaying phase (Phase II). Assume that TDMA is used in each phase and frame synchronization has been established. In each phase, each node is assigned a time slot to transmit its packet, which contains a large number of symbols. The source nodes take turns to exchange their information in Phase I. In Phase II, unlike the traditional cooperative communication, where the nodes repeat the received packets, each node transmits a new packet, whose symbols are generated by combing the received symbols from different nodes with its network coding vector.

Fig. 3 illustrates the differential space-time network code with the $k$ th symbols of the $N$ packets in one transmission period. In Phase I, each user node $U_{m}, m \in[1, N]$, is assigned a time slot $T_{m}$ to broadcast its packet to the rest of the user nodes with power $P_{I}$, where the $k$ th transmitted symbol is $v_{m}^{k}$. In Phase II, each node transmits a new packet with power $P_{I I}$, and the $k$ th transmitted symbols of the packets are $x_{n}^{k}$, $n \in[1, N]$. Let $P_{t}=P_{I}+P_{I I}$ be the total transmit power for a transmission. Assume that user nodes are far away from $D$, and the distance among the user nodes is small compared to the distance between the user nodes and $D$. A small $P_{I}$ is enough for the transmission in Phase I. Thus there is no direct link between the use nodes and the destination node in Phase I.
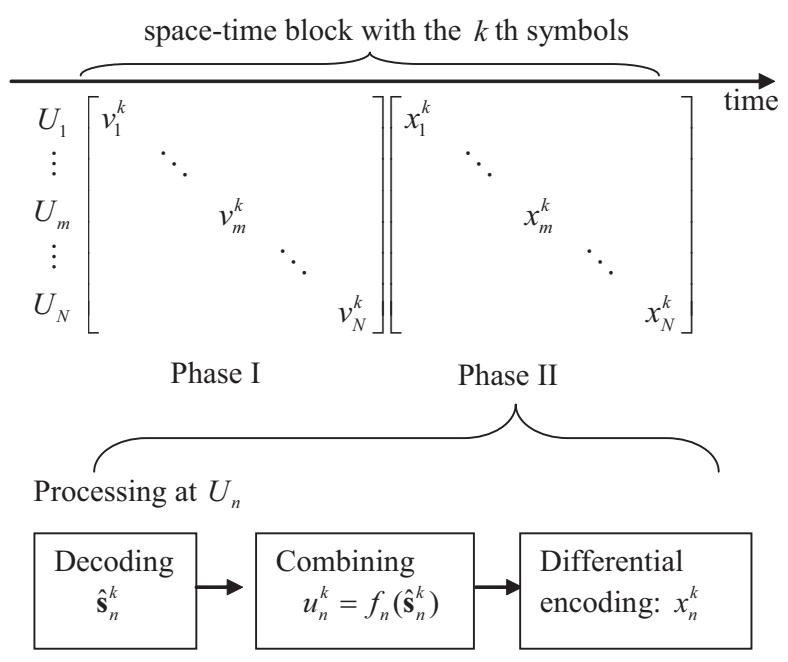

Fig. 3. The construction of the DSTNC with the $k$ th transmitted symbols of the $N$ packets for narrowband cooperative communications.

1) Signal Transmission in Phase I: Denote the $k$ th information symbols of the $N$ packets as a vector $\mathbf{s}^{k}=$ $\left[s_{1}^{k}, s_{2}^{k}, \ldots, s_{N}^{k}\right]^{\mathrm{T}}$ with $k \in\left[0, N_{s}-1\right]$, where $N_{s}$ is the number of information symbols in a packet. The information symbol $s_{m}^{k}$ of $U_{m}, m \in[1, N]$, is from an M-QAM constellation $\mathcal{A}$. Since no CSI is available at the user nodes, the transmitted symbol $v_{m}^{k}$ in Phase I is differentially encoded as $v_{m}^{k}=s_{m}^{k} \frac{v_{m}^{k-1}}{\left|v_{m}^{k-1}\right|}$ with $k=0$ denoting the initial reference symbol. The $k$ th transmitted symbol of $U_{m}$ received at $U_{n}$ in Phase I can be written as

$$
y_{m n}^{k}=\sqrt{P_{I}} g_{m n}^{k} v_{m}^{k}+w_{m n}^{k},
$$

where $w_{m n}^{k}$ is zero-mean and $N_{0}$-variance AWGN, $g_{m n}^{k}$ is the channel between $U_{m}$ and $U_{n}$ during the transmission of $v_{m}^{k}$. After decoding, $U_{n}$ obtains a set of estimated symbols $\hat{\mathbf{s}}_{n}^{k}=\left[\hat{s}_{1 n}^{k}, \ldots, \hat{s}_{m n}^{k}, \ldots, \hat{s}_{N n}^{k}\right]^{\mathrm{T}}$ from other nodes, where $\hat{s}_{m n}^{k}=$ $\beta_{m n} s_{m}^{k}$ with $\beta_{m n}$ indicating the detection state at $U_{n}$ for $s_{m}^{k}$. $\beta_{m n}$ for $m \neq n$ can be formulated as

$$
\beta_{m n}=\left\{\begin{array}{ll}
1, & \hat{s}_{m n}^{k}=s_{m}^{k} \\
0, & \hat{s}_{m n}^{k} \neq s_{m}^{k}
\end{array} .\right.
$$

It is obvious that $\beta_{n n}=1$ due to the fact that $U_{n}$ always has its own information. The detection states demonstrate that the user nodes just relay the symbols decoded correctly.

Then $U_{n}$ combines the elements of the estimation $\hat{\mathbf{s}}_{n}^{k}$ using a linear function to form a unique symbol as follows

$$
u_{n}^{k}=f_{n}\left(\hat{\mathbf{s}}_{n}^{k}\right)=\sum_{m=1}^{N} a_{m n} \hat{s}_{m n}^{k}=\mathbf{a}_{n}^{\mathrm{T}} \hat{\mathbf{s}}_{n}^{k}
$$

with $\mathbf{a}_{n}=\left[a_{1 n}, \ldots, a_{n n}, \ldots, a_{N n}\right]^{\mathrm{T}}$. Assume that $\mathbf{a}_{n}^{\mathrm{T}} \mathbf{a}_{n}=1$. We call the combining factors $a_{m n}, m, n \in[1, N]$, as network coding coefficients and $\mathbf{a}_{n}$ as the network coding vector at $U_{n}$.

2) Signal Transmission in Phase II: Because the destination does not have CSI, each user node would transmit the combined symbol $u_{n}^{k}$ differentially in Phase II, where $n \in[1, N], k \in\left[0, N_{s}-1\right]$. The nodes transmit their packets in their assigned time slots, and the $k$ th transmitted symbols 
of the $N$ packets can be written as a diagonal space-time matrix $\mathbf{D}_{x}^{k}=\operatorname{diag}\left[x_{1}^{k}, \ldots, x_{n}^{k}, \ldots, x_{N}^{k}\right]$, which is generated as follows

$$
\mathbf{D}_{x}^{k}=\mathbf{D}_{u}^{k} \mathbf{D}_{x}^{k-1} \overline{\mathbf{D}}_{x}^{k-1}
$$

where $\mathbf{D}_{x}^{k-1}=\operatorname{diag}\left[x_{1}^{k-1}, \ldots, x_{n}^{k-1}, \ldots, x_{N}^{k-1}\right]$ represents the space-time block with the $(k-1)$ th transmitted symbols of the $N$ packets, $\mathbf{D}_{u}^{k}=\operatorname{diag}\left[u_{1}^{k}, \ldots, u_{n}^{k}, \ldots, u_{N}^{k}\right]$ is the information block with the $k$ th combined symbols of the $N$ users, and $\overline{\mathbf{D}}_{x}^{k-1}=\operatorname{diag}\left[\frac{1}{\left|x_{1}^{k-1}\right|}, \ldots, \frac{1}{\left|x_{n}^{k-1}\right|}, \ldots, \frac{1}{\left|x_{N}^{k-1}\right|}\right]$ is the normalization block. Thus the $k$ th transmitted symbol of $U_{n}$ 's packet in Phase II is $x_{n}^{k}=u_{n}^{k} \frac{x_{n}^{k-1}}{\left|x_{n}^{k-1}\right|}$, which is differentially encoded between the combined symbol $u_{n}^{k}$ and the $(k-1)$ th symbol of the packet. The normalization block $\overline{\mathbf{D}}_{x}^{k-1}$ prevents the peak power of the transmitted signals from being too large or too small.

At the end of Phase II, $D$ has received the $N$ packets from the $N$ user nodes. The $k$ th signals of the received packets at $D$ can be written as

$$
\mathbf{y}^{k}=\sqrt{P_{I I}} \mathbf{D}_{x}^{k} \mathbf{h}^{k}+\mathbf{w}^{k},
$$

where $\mathbf{y}^{k}=\left[y_{1}^{k}, \ldots, y_{n}^{k}, \ldots, y_{N}^{k}\right]^{\mathrm{T}}, \quad \mathbf{w}^{k}=$ $\left[w_{1}^{k}, \ldots, w_{n}^{k}, \ldots, w_{N}^{k}\right]^{\mathrm{T}} \sim \mathcal{C N}\left(\mathbf{0}, \mathbf{I}_{N} N_{0}\right)$ is the received noise vector, $\mathbf{h}^{k}=\left[h_{1}^{k}, \ldots, h_{n}^{k}, \ldots, h_{N}^{k}\right]^{\mathrm{T}}$ with $h_{n}^{k}$ being the channel between $U_{n}$ and $D$ during the transmission of $x_{n}^{k}$. Assume that the channels do not change significantly over a period of two adjacent symbols within one packet, i.e. $h_{n}^{k}=h_{n}^{k-1}=h_{n}$ and $g_{m n}^{k}=g_{m n}^{k-1}=g_{m n}$. Thus we have $\mathbf{h}^{k}=\mathbf{h}^{k-1}=\mathbf{h}$, and the received signals can be expressed as

$$
\mathbf{y}^{k}=\mathbf{D}_{u}^{k} \overline{\mathbf{y}}^{k-1}+\tilde{\mathbf{w}}^{k}
$$

where $\overline{\mathbf{y}}^{k-1}=\overline{\mathbf{D}}_{x}^{k-1} \mathbf{y}^{k-1}$ and $\tilde{\mathbf{w}}^{k}=\mathbf{w}^{k}-\mathbf{D}_{u}^{k} \overline{\mathbf{D}}_{x}^{k-1} \mathbf{w}^{k-1}$. The equivalent noise vector $\tilde{\mathbf{w}}^{k}$ is zero-mean with the covariance matrix of

$$
\begin{aligned}
\boldsymbol{\Sigma}_{w} & =\mathrm{E}\left[\tilde{\mathbf{w}}^{k}\left(\tilde{\mathbf{w}}^{k}\right)^{\mathrm{H}}\right] \\
& =\left[\mathbf{I}_{N}+\left(\overline{\mathbf{D}}_{x}^{k-1}\right)^{2} \mathbf{D}_{u}^{k}\left(\mathbf{D}_{u}^{k}\right)^{\mathrm{H}}\right] N_{0}=\boldsymbol{\Lambda}_{w} N_{0},
\end{aligned}
$$

where $\boldsymbol{\Lambda}_{w}=\operatorname{diag}\left[\varepsilon_{1}, \ldots, \varepsilon_{n}, \ldots, \varepsilon_{N}\right]$ and $\varepsilon_{n}=1+\frac{\left|u_{n}^{k}\right|^{2}}{\left|x_{n}^{k-1}\right|^{2}}$.

\section{B. Signal Detection}

To make the relationship clear, substitute (2) and (3) into the received signals in (6) to get

$$
\mathbf{y}^{k}=\mathbf{S B A} \overline{\mathbf{y}}^{k-1}+\tilde{\mathbf{w}}^{k}
$$

where $\mathbf{S}=\operatorname{diag}\left[\mathbf{s}^{k^{\mathrm{T}}}, \ldots, \mathbf{s}^{k^{\mathrm{T}}}\right], \mathbf{A}=\operatorname{diag}\left[\mathbf{a}_{1}, \ldots, \mathbf{a}_{N}\right]$, and $\mathbf{B}=\operatorname{diag}\left[\beta_{11}, \ldots, \beta_{N 1}, \ldots, \beta_{1 N}, \ldots, \beta_{N N}\right]$.

Based on the received signal vector in (8), the maximum likelihood (ML) detection is used at $D$, and the estimation of $\mathbf{s}^{k}$, denoted as $\tilde{\mathbf{s}}^{k}$, can be written as

$$
\tilde{\mathbf{s}}^{k}=\arg \min _{\mathbf{s}^{k} \in \mathcal{A}^{N}}\left\{\left[\mathbf{y}^{k}-\mathbf{S A} \overline{\mathbf{y}}^{k-1}\right]^{\mathrm{H}} \boldsymbol{\Sigma}_{w}^{-1}\left[\mathbf{y}^{k}-\mathbf{S A} \overline{\mathbf{y}}^{k-1}\right]\right\} .
$$

As shown in (9), the decoding complexity of ML detection increases exponentially with the number of user nodes. Thus for large $N$ and/or large constellation size, sphere decoding method [21] [22] can be used to reduce the complexity. For high density nodes, they can be divided into subgroups and each group uses a DSTNC of smaller size to reduce the decoding complexity while sacrificing some diversity.
As for the detection at $U_{n}$ in Phase I, with the assumption that the channels do not change significantly over a period of two adjacent symbols, the received signal at $U_{n}$ from $U_{m}$ in Phase I becomes

$$
y_{m n}^{k}=\sqrt{P_{I}} g_{m n} v_{m}^{k}+w_{m n}^{k},
$$

and the ML detection for $s_{m}^{k}$ at $U_{n}$ is

$$
\hat{s}_{m n}^{k}=\arg \min _{s_{m} \in \mathcal{A}} \frac{\left|y_{m n}^{k}-s_{m} y_{m n}^{k-1}\right|^{2}}{\xi_{m} N_{0}},
$$

where $\xi_{m}=1+\frac{\left|s_{m}^{k}\right|^{2}}{\left|v_{m}^{k-1}\right|^{2}}$.

Note that in differential detection, because the receiver does not know the transmitted symbol, we can replace the signal terms in the variances of the equivalent noises with their means, i.e. $\varepsilon_{n}=1+\frac{E\left[\left|u_{n}^{k}\right|^{2}\right]}{\left|x_{n}^{k-1}\right|^{2}}$, and $\xi_{m}=1+\frac{E\left[\left|s_{m}^{k}\right|^{2}\right]}{\left|v_{m}^{k-1}\right|^{2}}$. Simulation results in Section $V$ show that this approximation leads to very small performance degradation. Also note that the detection states of the user nodes are not available at $D$, therefore in the ML detector (9), D assumes the user nodes can successfully decode. It is shown later by the simulations that the performances with and without knowledge of the detection states at $D$ are the same.

\section{PERFormanCE ANALYSis AND DESIGN OF DSTNC}

In this section, the performance of the DSTNC scheme is analyzed, and the PEP is derived. Based on the PEP analysis, we derive two design criteria for the network coding vectors, i.e., the diversity criterion and the product criterion.

\section{A. PEP Analysis}

The accurate performance analysis of a differential coded system requires the consideration of the quadratic receiving structure [23] [24]. We can well approximate the performance in high SNR situations by using an equivalent coherent receiver model (8) with $\overline{\mathbf{y}}^{k-1}$ acting as a known channel vector and $\tilde{\mathbf{w}}^{k}$ as the equivalent noise vector [25]. Since $\boldsymbol{\Sigma}_{w}$, the covariance matrix of $\tilde{\mathbf{w}}^{k}$, is clearly a diagonal matrix, the equivalent noise remains uncorrelated but with unequal variance entries. After normalized by $\boldsymbol{\Sigma}_{w}^{-\frac{1}{2}}$, which can be estimated at $D,(8)$ becomes

$$
\boldsymbol{\Sigma}_{w}^{-\frac{1}{2}} \mathbf{y}^{k}=\boldsymbol{\Sigma}_{w}^{-\frac{1}{2}} \mathbf{S B A} \overline{\mathbf{y}}^{k-1}+\overline{\mathbf{w}}^{k},
$$

where $\overline{\mathbf{w}}^{k}=\Sigma_{w}^{-\frac{1}{2}} \tilde{\mathbf{w}}^{k}$ has a covariance matrix $N_{0} \mathbf{I}_{N}$. The normalization just adjusts the signal strength at $D$ so that the noise power is uniform, and it does not affect the codeword error probability.

From the above equation, we can derive the PEP, i.e. the probability of transmitting $\mathbf{s}^{k}$ and deciding in favor of another $\mathbf{c}^{k}=\left[c_{1}^{k}, c_{2}^{k}, \ldots, c_{N}^{k}\right]^{\mathrm{T}}$ at the detector, conditioned by previously received signals and the detection states at the user nodes. The PEP is given by

$$
\begin{aligned}
& P\left(\mathbf{s}^{k} \rightarrow \mathbf{c}^{k} \mid \overline{\mathbf{y}}^{k-1},\left\{\beta_{11}, \ldots, \beta_{N 1}, \ldots, \beta_{1 N}, \ldots, \beta_{N N}\right\}\right) \\
& =Q\left(\sqrt{\frac{d^{2}(\mathbf{S}, \mathbf{C})}{2 N_{0}}}\right) \leq \exp \left(-\frac{d^{2}(\mathbf{S}, \mathbf{C})}{4 N_{0}}\right)
\end{aligned}
$$


where the inequality is the Chernoff bound [26], and $d^{2}(\mathbf{S}, \mathbf{C})$ is the distance between the received signals corresponding to respective codeword $\mathbf{s}^{k}$ and $\mathbf{c}^{k}$. The distance can be written as

$$
d^{2}(\mathbf{S}, \mathbf{C})=\left(\Delta \mathbf{S B A} \overline{\mathbf{y}}^{k-1}\right)^{\mathrm{H}} \boldsymbol{\Lambda}_{w}^{-1}\left(\Delta \mathbf{S B A} \overline{\mathbf{y}}^{k-1}\right),
$$

where $\Delta \mathbf{S}=\mathbf{S}-\mathbf{C}$ with $\mathbf{C}=\operatorname{diag}\left[\mathbf{c}^{k^{\mathrm{T}}}, \ldots, \mathbf{c}^{k^{\mathrm{T}}}\right]$. For moderate or high SNR scenarios, we make the following approximation $\mathbf{y}^{k-1}=\sqrt{P_{I I}} \mathbf{D}_{x}^{k-1} \mathbf{h}$, and the distance becomes

$$
d^{2}(\mathbf{S}, \mathbf{C})=P_{I I} \mathbf{h}^{\mathrm{H}}\left(\Delta \mathbf{S B A} \overline{\mathbf{D}}_{x}^{k-1} \mathbf{D}_{x}^{k-1}\right)^{\mathrm{H}} \boldsymbol{\Lambda}_{w}^{-1}\left(\Delta \mathbf{S B A} \overline{\mathbf{D}}_{x}^{k-1} \mathbf{D}_{x}^{k-1}\right) \mathbf{h} .
$$

Assuming that channels from different nodes to $D$ are spatially uncorrelated, averaging the bound in (13) with respect to $\mathbf{h}$ results in [26]

$$
\begin{aligned}
& P\left(\mathbf{s}^{k} \rightarrow \mathbf{c}^{k} \mid\left\{\beta_{11}, \ldots, \beta_{N 1}, \ldots, \beta_{1 N}, \ldots, \beta_{N N}\right\}\right) \\
& \leq \mathrm{E}_{\mathbf{h}}\left[\exp \left(-\frac{d^{2}(\mathbf{S}, \mathbf{C})}{4 N_{0}}\right)\right]=\prod_{n=1}^{N}\left(1+\frac{P_{I I} \lambda_{n}}{4 N_{0}}\right)^{-1},
\end{aligned}
$$

where $\lambda_{n}$ is the eigenvalue of the following $N \times N$ diagonal matrix

$$
\mathbf{R}_{h}^{\frac{1}{2}}\left(\Delta \mathbf{S B A} \overline{\mathbf{D}}_{x}^{k-1} \mathbf{D}_{x}^{k-1}\right)^{\mathrm{H}} \boldsymbol{\Lambda}_{w}^{-1}\left(\Delta \mathbf{S B A} \overline{\mathbf{D}}_{x}^{k-1} \mathbf{D}_{x}^{k-1}\right) \mathbf{R}_{h}^{\frac{1}{2}}
$$

with $\mathbf{R}_{h}=\operatorname{diag}\left[\sigma_{1}^{2}, \ldots, \sigma_{N}^{2}\right]$ being the correlation matrix of $\mathbf{h}$. It is easy to verify that the $n$th eigenvalue is $\lambda_{n}=$ $\frac{\sigma_{n}^{2}}{\varepsilon_{n}}\left|\sum_{m=1}^{N} \beta_{m n} a_{m n} \Delta s_{m}\right|^{2}$ with $\Delta s_{m}=s_{m}^{k}-c_{m}^{k}$.

Averaging with respect to the detection states of the user nodes, the PEP can be written as

$$
P\left(\mathbf{s}^{k} \rightarrow \mathbf{c}^{k}\right) \leq \mathrm{E}_{\beta_{m n}}\left[\prod_{n=1}^{N}\left(1+\frac{P_{I I} \lambda_{n}}{4 N_{0}}\right)^{-1}\right] .
$$

Because the signal detection at each user is independent and the detection of symbols from different sources at a certain user is also independent, the order of expectation operator and production operator in (18) can be exchanged as follows

$$
P\left(\mathbf{s}^{k} \rightarrow \mathbf{c}^{k}\right) \leq \prod_{n=1}^{N} \mathrm{E}_{\beta_{m n}}\left[\left(1+\frac{P_{I I} \sigma_{n}^{2}\left|\sum_{m=1}^{N} \beta_{m n} a_{m n} \Delta s_{m}\right|^{2}}{4 N_{0} \varepsilon_{m}}\right)^{-1}\right] .
$$

Assuming that the SER at $U_{n}$ for the symbols from $U_{m}$ is $p_{m n}, \beta_{m n}$ 's are independent Bernoulli random variables with a distribution

$$
P\left(\beta_{m n}\right)= \begin{cases}1-p_{m n}, & \text { for } \beta_{m n}=1 \\ p_{m n}, & \text { for } \beta_{m n}=0\end{cases}
$$

Considering all possibilities of the detection state $\left\{\beta_{11}, \ldots, \beta_{N 1}, \ldots, \beta_{1 N}, \ldots, \beta_{N N}\right\}$, the PEP can be written as (21) which is shown on the top of this page. The first term in (21) represents the case when all symbols received at $U_{n}$ are decoded correctly, the second term represents the case when one of the symbols received at $U_{n}$ is decoded incorrectly, and so on. When SNR is high, SERs are small and the higher order terms of SERs could be ignored. Thus the PEP can be approximated by (22) on the top of this page, where H.O.T. stands for the higher order terms.
Diversity is an important criterion since it determines the slop of the performance curve. However, it is obvious from (22) that $\left|\mathbf{a}_{n}^{\mathrm{T}} \Delta \mathbf{s}\right|=0$ would result in the loss of diversity. In order to achieve the maximum diversity, the first criterion of the network coding vectors is derived as follows.

Diversity criterion: The full diversity gain $N$ can be achieved if the following maximum diversity condition holds true for any distinct pair $\left\{\mathbf{s}^{k}, \mathbf{c}^{k}\right\}$,

$$
\left|\mathbf{a}_{n}^{\mathrm{T}} \Delta \mathbf{s}\right| \neq 0, \quad \forall n \in[1, N], \forall \mathbf{s}^{k}, \mathbf{c}^{k} \in \mathcal{A}^{N} .
$$

Theorem 1: Let $d_{\max }$ represent the maximum Euclidean distance of $\mathcal{A}$. If $\forall n \in[1, N],\left|\mathbf{a}_{n}^{\mathrm{T}} \Delta \mathbf{s}\right| \neq 0$ holds true for any distinct pair $\left\{\mathbf{s}^{k}, \mathbf{c}^{k}\right\}$, the PEP under high SNR is upper bounded by

$$
P\left(\mathbf{s}^{k} \rightarrow \mathbf{c}^{k}\right)<G\left(\frac{P_{t}}{N_{0}}\right)^{-N}
$$

where $G$ is coding advantage and

$$
G=\prod_{n=1}^{N} \frac{1}{\left|\mathbf{a}_{n}^{\mathrm{T}} \Delta \mathbf{s}\right|^{2}}\left(\frac{4 \varepsilon_{n}}{(1-\alpha) \sigma_{n}^{2}}+\sum_{l=1, l \neq n}^{N} \frac{N C_{M} \xi_{l} d_{\max }^{2}}{\alpha \sigma_{l n}^{2}}\right)
$$

with $\alpha=\frac{P_{I}}{P_{t}}$. $C_{M}$ is constant for a certain constellation.

Proof: See Appendix.

It can be seen from (24) that, for a fixed number of user nodes, the adopted signal constellation, the power allocation $\alpha$ and channel conditions can influence the coding advantage $G$ but not the diversity order. When given the constellation, $\alpha$ and channel conditions, the PEP depends on the design of network coding vectors. Thus according to Theorem 1, the second design criterion is derived as follows to get a better performance.

Product criterion: The minimum value of the product $\prod_{n=1}^{N}\left|\mathbf{a}_{n}^{\mathrm{T}} \Delta \mathbf{s}\right|^{2}$ over all pairs of distinct signal vectors $\mathbf{s}^{k}$ and $\mathbf{c}^{k}$ should be as large as possible.

The product criterion is of secondary importance and should be optimized if full diversity is achieved.

\section{B. Design of DSTNC}

Let $\mathbf{A}_{N}$ denote the combining matrix (network coding matrix), which is constructed from the network coding vectors of the $N$ user nodes as follows

$$
\mathbf{A}_{N}^{\mathrm{T}}=\left[\begin{array}{lllll}
\mathbf{a}_{1} & \cdots & \mathbf{a}_{n} & \cdots & \mathbf{a}_{N}
\end{array}\right] .
$$

Let $\mathbf{s}_{f}=\mathbf{A}_{N} \mathbf{s}$, where $\mathbf{s}=\left[s_{1}, \ldots, s_{N}\right]^{\mathrm{T}} \in \mathcal{A}^{N}$. According to the diversity criterion, $\mathbf{A}_{N}$ should be designed to satisfy the following property: the original signal constellation is rotated and expanded such that there is no zero entry in the vector $\mathbf{s}_{f}$ for any $\mathbf{s} \neq \mathbf{0}_{N}$. This is referred to as the signal space diversity technique [27] [28]. According to the product criterion, the minimum product distance of a set of signal points should be maximized, which is also investigated in [27], [28]. This diversity technique has been applied to MIMO coding schemes such as [29], [30]. Note that the combining matrices can be constructed based on Hadamard matrices and Vandermonde matrices. However, the combing matrices based on Vandermonde matrices result in larger minimum product distance than those based on Hadamard matrices [30]. A combining matrix based on Vandermonde matrices with parameters $\theta_{1}, \theta_{2}, \ldots, \theta_{N}$ can be written as 


$$
\begin{aligned}
& P\left(\mathbf{s}^{k} \rightarrow \mathbf{c}^{k}\right) \leq \prod_{n=1}^{N}\left[\frac{1}{1+\frac{P_{I I} \sigma_{n}^{2}}{4 N_{0} \varepsilon_{n}}\left|\mathbf{a}_{n}^{\mathrm{T}} \Delta \mathbf{s}\right|^{2}} \prod_{m=1, m \neq n}^{N}\left(1-p_{m n}\right)\right. \\
& \left.+\sum_{l=1, l \neq n}^{N}\left(\frac{1}{1+\frac{P_{I I} \sigma_{n}^{2}}{4 N_{0} \varepsilon_{n}}\left|\sum_{m=1, m \neq l}^{N} a_{m n} \Delta s_{m}\right|^{2}} \prod_{i=1, i \neq l, i \neq n}^{N}\left(1-p_{i n}\right) p_{l n}\right)+\cdots+\frac{1}{1+\frac{P_{I I} \sigma_{n}^{2}}{4 N_{0} \varepsilon_{n}}\left|a_{n n} \Delta s_{n}\right|^{2}} \prod_{m=1, m \neq n}^{N} p_{m n}\right],
\end{aligned}
$$

$$
\begin{aligned}
P\left(\mathbf{s}^{k} \rightarrow \mathbf{c}^{k}\right) & \leq \prod_{n=1}^{N}\left[\frac{1}{1+\frac{P_{I I} \sigma_{n}^{2}}{4 N_{0} \varepsilon_{n}}\left|\mathbf{a}_{n}^{\mathrm{T}} \Delta \mathbf{s}\right|^{2}}\left(1-\sum_{m=1, m \neq n}^{N} p_{m n}\right)+\sum_{l=1, l \neq n}^{N} \frac{1}{1+\frac{P_{I I} \sigma_{n}^{2}}{4 N_{0} \varepsilon_{n}}\left|\sum_{m=1, m \neq l}^{N} a_{m n} \Delta s_{m}\right|^{2}} p_{l n}+\text { H.O.T. }\right] \\
& \leq \prod_{n=1}^{N}\left[\frac{1}{1+\frac{P_{I I} \sigma_{n}^{2}}{4 N_{0} \varepsilon_{n}}\left|\mathbf{a}_{n}^{\mathrm{T}} \Delta \mathbf{s}\right|^{2}}\left(1-\sum_{m=1, m \neq n}^{N} p_{m n}\right)+\sum_{l=1, l \neq n}^{N} p_{l n}+\text { H.O.T. }\right],
\end{aligned}
$$

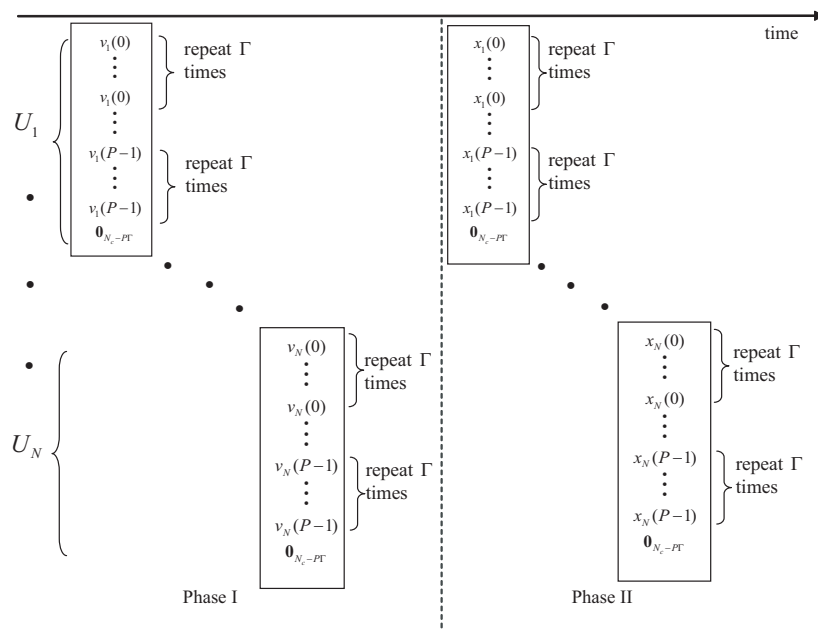

Fig. 4. The construction of DSTFNC for broadband cooperative communications.

$$
\mathbf{A}_{N}^{\mathrm{T}}\left(\theta_{1}, \theta_{2}, \ldots, \theta_{N}\right)=\frac{1}{\sqrt{N}}\left[\begin{array}{cccc}
1 & 1 & \cdots & 1 \\
\theta_{1} & \theta_{2} & \cdots & \theta_{N} \\
\vdots & \vdots & \ddots & \vdots \\
\theta_{1}^{N-1} & \theta_{2}^{N-1} & \cdots & \theta_{N}^{N-1}
\end{array}\right]
$$

Some best known transform matrices $\mathbf{A}_{N}^{\mathrm{T}}$ based on Vandermonde matrices are summarized in [30]. More constructions and details of the transform matrices can be found in [27], [28].

Based on the above description, the network coding vector for $U_{n}$ is

$$
\mathbf{a}_{n}=\frac{1}{\sqrt{N}}\left[\begin{array}{llll}
1 & \theta_{n} & \cdots & \theta_{n}^{N-1}
\end{array}\right]^{\mathrm{T}},
$$

where $\theta_{n}$ is determined by the number of nodes and the construction method. This means each node can independently generate its combining function according to the assigned parameter $\theta_{n}, n \in[1, N]$. With these network coding vectors, the proposed DSTNC scheme can achieve full diversity, which equals the number of user nodes, without the requirement of synchronization or channel estimation.

\section{DESIGN OF DSTFNC FROM DSTNC}

In this section we consider the differential transmission for broadband multi-source cooperative communications. The channels in the network shown in Fig. 2 are assumed frequency selective fading. Assume that the numbers of multipaths between any pair of user nodes and between the user nodes and $D$ are $L_{1}$ and $L_{2}$, respectively. The channel frequency response from $U_{m}$ to $U_{n}$ is given by $G_{m n}(q)=$ $\sum_{l=0}^{L_{1}-1} g_{m n}(l) e^{-j \frac{2 \pi q \tau_{m n}(l)}{T}}$, where $q \in\left[0, N_{c}-1\right], g_{m n}(l)$ and $\tau_{m n}(l)$ are the complex amplitude and delay of the $l$ th path, respectively, and $T$ is the OFDM symbol period. The channel coefficients $g_{m n}(l)$ 's are modeled as zero-mean complex Gaussian variables with variances $\mathrm{E}\left|g_{m n}(l)\right|^{2}=\sigma_{m n, l}^{2} \cdot g_{m n}(l)$ for different $m, n \in[1, N]$ and $l \in\left[0, L_{1}-1\right]$ are assumed independent, and the total power is $\sum_{l=0}^{L_{1}-1} \sigma_{m n, l}^{2}=\sigma_{m n}^{2}$. The channel frequency response from $U_{m}$ to $D$ is given by $H_{m}(q)=\sum_{l=0}^{L_{2}-1} h_{m}(l) e^{-j \frac{2 \pi q \tau_{m}(l)}{T}}$, where $h_{m}(l)$ and $\tau_{m}(l)$ are the complex amplitude and delay of the $l$ th path, respectively. The channel coefficients $h_{m}(l)$ 's are modeled as zero-mean complex Gaussian variables with variances $\mathrm{E}\left|h_{m}(l)\right|^{2}=\sigma_{m, l}$ and $h_{m}(l)$ for different $m \in[1, N]$ and $l \in\left[0, L_{2}-1\right]$ are assumed independent. The total power is normalized such that $\sum_{l=0}^{L_{2}-1} \sigma_{m, l}^{2}=\sigma_{m}^{2}$. TDMA is still used in Phase I and Phase II to overcome the imperfect synchronization issue. Each user node employs an OFDM modulator with $N_{c}$ subcarriers. Based on the proposed DSTNC scheme, a differential encoding scheme in frequency domain within each OFDM block is designed.

\section{A. Distributed DSTFNC From DSTNC}

The distributed DSTFNC scheme is motivated by the differential space-frequency modulation scheme in [20]. Full diversity space frequency codes for MIMO-OFDM systems are obtained from space time codes via mapping in [31]. In this subsection, we will generate the DSTFNC for broadband multi-source cooperative communication systems by mapping from the proposed DSTNC.

The two phases of a transmission is illustrated in Fig. 4, which has a similar structure to Fig. 3. The OFDM block for each user is divided into $P$ groups in which each transmitted symbol is repeated $\Gamma$ times, where $1 \leq \Gamma \leq L_{2}$ and $P=$ $\left\lfloor\frac{N_{c}}{\Gamma}\right\rfloor$, i.e., the largest integer not greater than $\frac{N_{c}}{\Gamma}$. The integer $\Gamma$ is adjustable for different diversities. $p=0$ is the reference group for the differential coding.

1) Differential Transmission in Phase I: The $p$ th group in the $m$ th OFDM period received at $U_{n}$ in Phase I can be written 


$$
\mathbf{y}_{m n}^{p}=\sqrt{P_{I}} \mathbf{1}_{\Gamma} \otimes v_{m}(p) \mathbf{G}_{m n}^{p}+\mathbf{w}_{m n}^{p},
$$

where $\mathbf{y}_{m n}^{p}=\left[y_{m n}\left(k_{p, 1}\right), \ldots, y_{m n}\left(k_{p, \Gamma}\right)\right]^{\mathrm{T}}$ with $y_{m n}\left(k_{p, l}\right)$ being the signal received from $U_{m}$ on the $k_{p, l}$ th subcarrier, and $\mathbf{w}_{m n}^{p}=\left[w_{m n}\left(k_{p, 1}\right), \ldots, w_{m n}\left(k_{p, \Gamma}\right)\right]^{\mathrm{T}}$ with $w_{m n}\left(k_{p, \gamma}\right)$ being the zero-mean and $N_{0}$-variance AWGN on the $k_{p l}$ th subcarrier received from $U_{m}$ at $U_{n}$, and $\mathbf{G}_{m n}^{p}=$ $\left[G_{m n}\left(k_{p, 1}\right), \ldots, G_{m n}\left(k_{p, \Gamma}\right)\right]^{\mathrm{T}}$. As in Section II. A, the transmitted symbol is $v_{m}(p)=s_{m}(p) \frac{v_{m}(p-1)}{\left|v_{m}(p-1)\right|}$, where $s_{m}(p)$ is the $p$ th information symbol of $U_{m}$. Note that the processing is the same as that for frequency flat fading except that the differential modulation is performed over subcarriers.

After decoding in Phase I, $U_{n}$ obtains a set of estimations $\hat{\mathbf{S}}_{n}=\left[\hat{\mathbf{s}}_{1 n}, \ldots, \hat{\mathbf{s}}_{m n}, \ldots, \hat{\mathbf{s}}_{N n}\right]^{\mathrm{T}}$ from other users, where $\hat{\mathbf{s}}_{m n}=\left[\hat{s}_{m n}(0), \hat{s}_{m n}(1), \ldots, \hat{s}_{m n}(P-1)\right]^{\mathrm{T}}$ is the estimated signal vector from $U_{m}$. According to (3), the combined symbol generated at $U_{n}$ for $p=0,1, . . P-1$ is

$$
u_{n}(p)=f_{n}\left(\hat{\mathbf{s}}_{n}(p)\right)=\sum_{m=1}^{N} a_{m n} \hat{s}_{m n}(p)=\mathbf{a}_{n}^{\mathrm{T}} \hat{\mathbf{s}}_{n, p}
$$

where $\hat{\mathbf{s}}_{n, p}=\left[\hat{s}_{1 n}(p), \hat{s}_{2 n}(p), \ldots, \hat{s}_{N n}(p)\right]^{\mathrm{T}}$ and $\mathbf{a}_{n}$ is the coding vector designed in Section III.

2) Differential Transmission in Phase II: Because $D$ does not have any kind of CSI, the users transmit their $p$ th combined symbols $\mathbf{u}_{p}=\left[u_{1}(p), u_{2}(p), \ldots, u_{N}(p)\right]$ differentially. Let $\mathbf{X}_{p}$ denote the $p$ th group of the DSTFNC, which can be formulated as

$$
\mathbf{X}_{p}=\mathbf{1}_{\Gamma} \otimes \mathbf{D}_{x}^{p},
$$

where $\mathbf{D}_{x}^{p}=\operatorname{diag}\left[x_{1}(p), x_{2}(p), \ldots, x_{N}(p)\right]$ with $x_{n}(p)$ being the transmitted symbol in the $p$ th group of the OFDM symbol from $U_{n} . \mathbf{D}_{x}^{p}$ is differentially encoded according to (4),

$$
\mathbf{D}_{x}^{p}=\mathbf{D}_{u}^{p} \mathbf{D}_{x}^{p-1} \overline{\mathbf{D}}_{x}^{p-1},
$$

where $\mathbf{D}_{u}^{p}=\operatorname{diag}\left[u_{1}(p), u_{2}(p), \ldots, u_{N}(p)\right], \quad \mathbf{D}_{x}^{p-1}=$ $\operatorname{diag}\left[x_{1}(p-1), x_{2}(p-1), \ldots, x_{N}(p-1)\right]$, and $\overline{\mathbf{D}}_{x}^{p-1}=$ $\operatorname{diag}\left[\frac{1}{\left.\mid x_{1}(p-1)\right]}, \frac{1}{\left|x_{2}(p-1)\right|}, \ldots, \frac{1}{\left|x_{N}(p-1)\right|}\right]$. Obviously, $\mathbf{D}_{x}^{p}$ is the DSTNC in Section II. The difference is that the DSTNC is differentially encoded in time domain while the DSTFNC is in frequency domain. Ignoring the zeros filled in Fig. 4(b) when $N_{c}$ is not an integer multiple of $N \Gamma$, the DSTFNC can be written as a concatenation of the $P$ groups

$$
\mathbf{X}=\left[\mathbf{X}_{0}^{\mathrm{T}}, \mathbf{X}_{1}^{\mathrm{T}}, \ldots, \mathbf{X}_{P-1}^{\mathrm{T}}\right]^{\mathrm{T}} \text {. }
$$

Let $\mathbf{D}_{X_{p}}=\operatorname{diag}\left[x_{1}(p), \ldots, x_{N}(p), \ldots, x_{1}(p), \ldots, x_{N}(p)\right]$ denote the diagonal version of $\mathbf{X}_{p}$. Then the $p$ th group of the received signals at $D$ can be written as

$$
\mathbf{y}^{p}=\sqrt{P_{I I}} \mathbf{D}_{X_{p}} \mathbf{H}^{p}+\mathbf{w}^{p},
$$

where $\mathbf{H}^{p}=\left[H_{1}\left(k_{p, 1}^{(1)}\right), \ldots, H_{N}\left(k_{p, 1}^{(N)}\right), \ldots, H_{1}\left(k_{p, \Gamma}^{(1)}\right), \ldots, H_{N}\left(k_{p, \Gamma}^{(N)}\right)\right]^{\mathrm{T}}$ is the $p$ th group of channel vector with $k_{p, \gamma}^{(m)} \in\left[0, N_{c}-1\right]$ being the index of subcarrier on which $x_{m}(p)$ is transmitted by $U_{m}, \mathbf{y}^{p}=$ $\left[y_{1}\left(k_{p, 1}^{(1)}\right), \ldots, y_{N}\left(k_{p, 1}^{(N)}\right), \ldots, y_{1}\left(k_{p, \Gamma}^{(1)}\right), \ldots, y_{N}\left(k_{p, \Gamma}^{(N)}\right)\right]^{\mathrm{T}}$

is the $p$ th group of the received signal vector with $y_{m}\left(k_{p, \gamma}^{(m)}\right)$ being the signal received from $U_{m}$ on the $k_{p, \gamma}^{(m)}$ th subcarrier, and $\mathbf{w}^{p}=$ $\left[w_{1}\left(k_{p, 1}^{(1)}\right), \ldots, w_{N}\left(k_{p, 1}^{(N)}\right), \ldots, w_{1}\left(k_{p, \Gamma}^{(1)}\right), \ldots, w_{N}\left(k_{p, \Gamma}^{(N)}\right)\right]^{\mathrm{T}}$ with $w_{m}\left(k_{p, \gamma}^{(m)}\right)$ being the zero-mean AWGN with variance $N_{0}$ received by $D$ on the $k_{p, \gamma}^{(m)}$ th subcarrier during the $m$ th OFDM period in Phase II. It can be seen from (30)-(32) that the signals are differentially encoded over groups of subcarriers. Define $\boldsymbol{\Delta}_{h, p}=\mathbf{H}_{p}-\mathbf{H}_{p-1}$ as the channel difference between $\mathbf{H}_{p}$ and $\mathbf{H}_{p-1}$. Since $\mathbf{y}^{p}=\sqrt{P_{I I}} \mathbf{D}_{X_{p}} \mathbf{H}^{p}+\mathbf{w}^{p}$ and $\mathbf{D}_{X_{p}}=\mathbf{I}_{\Gamma} \otimes\left(\mathbf{D}_{u}^{p} \overline{\mathbf{D}}_{x}^{p-1}\right) \mathbf{D}_{X_{p-1}}$, we have

$$
\mathbf{y}^{p}=\mathbf{I}_{\Gamma} \otimes\left(\mathbf{D}_{u}^{p} \overline{\mathbf{D}}_{x}^{p-1}\right) \mathbf{y}^{p-1}+\sqrt{P_{I I}} \mathbf{D}_{X_{p}} \boldsymbol{\Delta}_{h, p}+\tilde{\mathbf{w}}^{p}
$$

where $\tilde{\mathbf{w}}^{p}=\mathbf{w}^{p}-\mathbf{I}_{\Gamma} \otimes\left(\mathbf{D}_{u}^{p} \overline{\mathbf{D}}_{x}^{p-1}\right) \mathbf{w}^{p-1}$. The equivalent noise $\tilde{\mathbf{W}}^{p}$ is zero-mean and the variance is

$$
\begin{aligned}
\boldsymbol{\Sigma}_{w}^{\prime} & =\mathrm{E}\left[\tilde{\mathbf{w}}^{p}\left(\tilde{\mathbf{w}}^{p}\right)^{\mathrm{H}}\right] \\
& =\mathbf{I}_{\Gamma} \otimes\left[\mathbf{I}_{N}+\left(\overline{\mathbf{D}}_{x}^{p-1}\right)^{2} \mathbf{D}_{u}^{p}\left(\mathbf{D}_{u}^{p}\right)^{\mathrm{H}}\right] N_{0}=\boldsymbol{\Lambda}_{w}^{\prime} N_{0} .
\end{aligned}
$$

It is obvious that $\boldsymbol{\Lambda}_{w}^{\prime}=\mathbf{I}_{\Gamma} \otimes \boldsymbol{\Lambda}_{w}$, where $\boldsymbol{\Lambda}_{w}$ is defined in (7).

\section{B. Decoding and Channel Permutation}

1) Decoding at $D$ in Phase II: We consider differential decoding over two adjacent received groups $\mathbf{y}^{p}$ and $\mathbf{y}^{p-1}$ for any $p=1,2, \ldots P-1$, where $p=0$ is the reference group. Let $\mathbf{s}_{p}=\left[s_{1}(p), s_{2}(p), \ldots, s_{N}(p)\right]^{\mathrm{T}}$ denote the $p$ th information symbols from the $N$ user nodes and $\mathbf{S}=\operatorname{diag}\left[\mathbf{s}_{p}^{\mathrm{T}}, \ldots, \mathbf{s}_{p}^{\mathrm{T}}\right]$. If $\mathbf{H}^{p} \approx \mathbf{H}^{p-1}$, or $\left\|\boldsymbol{\Delta}_{h, p}\right\|_{F}$ is small enough such that $\sqrt{P_{I I}} \|$ $\boldsymbol{\Delta}_{h, p} \|_{F}$ is much less than $\left\|\tilde{\mathbf{w}}_{p}\right\|_{F}$ [20], then a near ML decoding can be performed as (36) on the top of this page. When $\mathrm{N}$ and/or constellation size is large, sphere decoder [21] [22] can be used to reduce the decoding complexity.

2) Channel Permutation at Each User Node in Phase II: When the delay spread is small with respect to the OFDM period, the channel frequency response changes slowly over two adjacent subcarriers. In this case, the subcarrier indices $k_{p, \gamma}^{(m)}$ and $k_{p-1, \gamma}^{(m)}$ for $U_{m}, m \in[1, N]$, can be chosen as the two adjacent subcarriers of $\mathbf{H}_{m}=\left[H_{m}(0), H_{m}(1), \ldots, H_{m}\left(N_{c}-\right.\right.$ $1)$. When the delay spread is large with respect to the OFDM period, the channel frequency response changes rapidly. In this case, when the statistical channel PDP (not exact CSI) from $U_{n}$ to $D$ is known at $U_{n}$, we can permute the subcarriers to get smooth logical channels so that the differential decoding can be performed successfully. Because the permutation optimization by maximizing the SER or bit error rate (BER) is intractable, we consider an permutation approach of maximizing the average SNR as in [20].

Define the average SNR as

$$
\mathrm{SNR}_{a v}=\frac{\sum_{p=1}^{P-1}\left(\mathrm{E}\left\|\mathbf{I}_{\Gamma} \otimes\left(\mathbf{D}_{u}^{p} \overline{\mathbf{D}}_{x}^{p-1}\right) \mathbf{y}^{p-1}\right\|_{\mathrm{F}}^{2}\right)}{\sum_{p=1}^{P-1}\left(\mathrm{E}\left\|\sqrt{P_{I I}} \mathbf{D}_{X_{p}} \cdot \boldsymbol{\Delta}_{h, p}+\tilde{\mathbf{w}}^{p}\right\|_{\mathrm{F}}^{2}\right)} \text {. }
$$

We try to minimize $\sum_{p=1}^{P-1}\left(\mathrm{E}\left\|\sqrt{P_{I I}} \mathbf{D}_{X_{p}} \boldsymbol{\Delta}_{h, p}+\tilde{\mathbf{w}}\right\|_{\mathrm{F}}^{2}\right)$ to maximize $\mathrm{SNR}_{a v}$. Because the noise, the signals, and the channels are independent with each other, we can simplify the minimization problem as (38) on the top of this page, where $\Phi_{n}=\sum_{p=1}^{P-1}\left(\mathrm{E}\left|x_{n}(p)\right|^{2} \mathrm{E} \sum_{\gamma=1}^{\Gamma}\left|H_{n}\left(k_{p, \gamma}^{(n)}\right)-H_{n}\left(k_{p-1, \gamma}^{(n)}\right)\right|^{2}\right)$. $\mathrm{E}\left|x_{n}(p)\right|^{2}$ is a constant related to the signal constellation, so we will omit it in the following. It can be seen from (38) that the minimization problem can be solved by minimizing $\Phi_{n}$ independently at each user node. Therefore, the permutation approach for a certain user node $U_{n}$ is considered and the other nodes can permute their subcarriers in the same way. 


$$
\tilde{\mathbf{s}}_{p}=\arg \min _{\mathbf{s}_{p} \in \mathcal{A}^{N}}\left\{\left[\mathbf{y}^{p}-\mathbf{I}_{\Gamma} \otimes\left(\mathbf{S A} \overline{\mathbf{D}}_{x}^{p-1}\right) \mathbf{y}^{p-1}\right]^{\mathrm{H}}\left(\boldsymbol{\Sigma}_{w}^{\prime}\right)^{-1}\left[\mathbf{y}^{p}-\mathbf{I}_{\Gamma} \otimes\left(\mathbf{S A} \overline{\mathbf{D}}_{x}^{p-1}\right) \mathbf{y}^{p-1}\right]\right\}
$$

$$
\begin{aligned}
\min \sum_{p=1}^{P-1}\left(\mathrm{E}\left\|\sqrt{P_{I I}} \mathbf{D}_{X_{p}} \boldsymbol{\Delta}_{h, p}\right\|_{\mathrm{F}}^{2}\right) & =\min \sum_{p=1}^{P-1}\left(P_{I I} \sum_{n=1}^{N}\left(\mathrm{E}\left|x_{n}(p)\right|^{2} \mathrm{E} \sum_{\gamma=1}^{\Gamma}\left|H_{n}\left(k_{p, \gamma}^{(n)}\right)-H_{n}\left(k_{p-1, \gamma}^{(n)}\right)\right|^{2}\right)\right) \\
& =\min P_{I I} \sum_{n=1}^{N} \Phi_{n}
\end{aligned}
$$

$$
\begin{aligned}
\Phi_{n} & =\mathrm{E} \sum_{p=1}^{P-1} \sum_{\gamma=1}^{\Gamma}\left|\sum_{l=0}^{L_{2}-1} h_{n}(l) e^{-j \frac{2 \pi k_{p, \gamma}^{(n)} \tau_{n}(l)}{T}}-\sum_{l=0}^{L_{2}-1} h_{n}(l) e^{-j \frac{2 \pi k_{p-1, \gamma}^{(n)} \tau_{n}(l)}{T}}\right|^{2} \\
& =\sum_{p=1}^{P-1} \sum_{\gamma=1}^{\Gamma} \sum_{l=0}^{L_{2}-1} 4 \sigma_{n, l}^{2} \sin ^{2}\left[\frac{\tau_{n}(l)\left(k_{p, \gamma}^{(n)}-k_{p-1, \gamma}^{(n)}\right)}{T}\right] .
\end{aligned}
$$

For $U_{n}, \Phi_{n}$ can be written as (39), which is on the top of this page. Let $d(i, j)=\sum_{l=0}^{L_{2}-1} 4 \sigma_{n, l}^{2} \sin ^{2}\left[\frac{\tau_{n}(l)(i-j)}{T}\right]$. Then $\Phi_{n}$ becomes

$$
\Phi_{n}=\sum_{p=1}^{P-1} \sum_{\gamma=1}^{\Gamma} d\left(k_{p, \gamma}^{(n)}, k_{p-1, \gamma}^{(n)}\right) .
$$

The minimization of $\Phi_{n}$ in (40) is the shortest-path problem [32] and can be solved by using the idea of Dijkstra's algorithm [20], [32] as follows.

- Let $V_{0}=\{1,2, \ldots, P \Gamma\}$. Randomly choose $\Gamma$ elements $\left\{k_{0,1}, \ldots, k_{0, \Gamma}\right\}$ from $V_{0}$ to form a set $E_{0}$, and let the rest elements be $V_{1}=V_{0}-E_{0}$.

- For $\mathrm{p}=1: \mathrm{P}-1$

1) Choose $\Gamma$ elements $\left\{k_{p, 1}, \ldots, k_{p, \Gamma}\right\}$ from $V_{p}$ to form a set $E_{p}$ whose elements are nearest to the set $E_{p-1}$, i.e., to minimize $\sum_{\gamma=1}^{\Gamma} d\left(k_{p, \gamma}, k_{p-1, \gamma}\right)$.

2) Denote the set of the rest elements as $V_{p+1}=V_{p}$ $E_{p}$.

By independently minimizing $\Phi_{n}, n \in[1, N]$, each user can get a smooth logical channel.

3) Decoding and Channel Permutation in Phase I: As for the signal detection in Phase I, differential decoding is performed. Define $\boldsymbol{\Delta}_{g, p}=\mathbf{G}_{m n}^{p}-\mathbf{G}_{m n}^{p-1}$ as the channel difference between $\mathbf{G}_{m n}^{p}$ and $\mathbf{G}_{m n}^{p-1}$. Assuming that $\mathbf{G}_{m n}^{p} \approx$ $\mathbf{G}_{m n}^{p-1}$, or $\left\|\boldsymbol{\Delta}_{g, p}\right\|_{\mathrm{F}}$ is small enough, a near ML detection for $s_{m}(p), p \in[0, P-1]$, can be written as

$\hat{s}_{m n}(p)=\arg \min _{s_{m} \in \mathcal{A}} \sum_{\gamma=1}^{\Gamma} \frac{\left|y_{m n}^{p}\left(k_{p, \gamma}\right)-s_{m} y_{m n, p-1}\left(k_{p-1, \gamma}\right)\right|^{2}}{\xi_{m}^{\prime} N_{0}}$,

where $\xi_{m}^{\prime}=1+\frac{\mathrm{E}\left|s_{m}(p)\right|^{2}}{\left|v_{m}(p-1)\right|^{2}}$. To decode successfully for different delay spreads, the sets of the subcarriers can be permutated through the same way described above.

\section{Simulation Results}

In this section, we present simulation results of the DSTNC and the STFNC for narrow-band and broadband wireless multi-source cooperative communications, respectively. Various numbers of user nodes are used with $N=2,3$, and 4 . The corresponding network coding vectors are generated

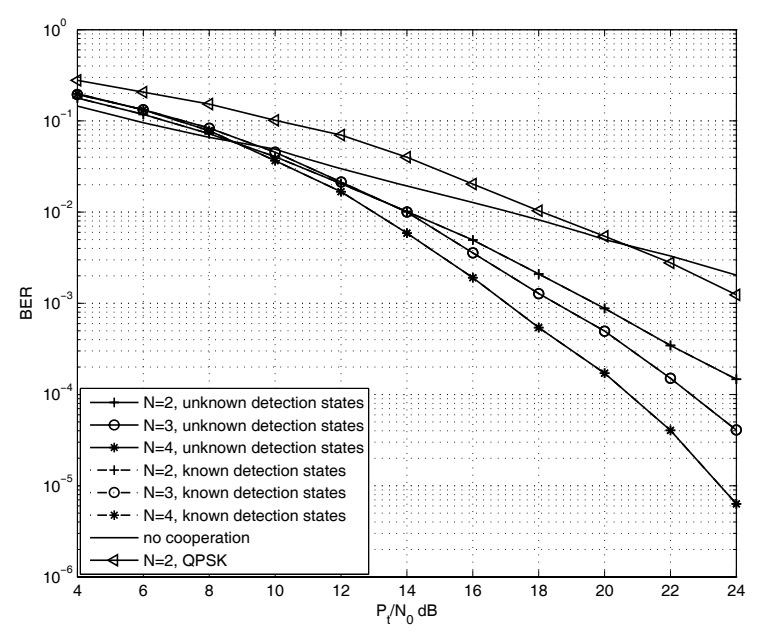

Fig. 5. BER performance of DSTNC for different number of user nodes with $d_{u}=d / 3$.

according to (27). For $N=2, \theta_{n}=e^{j \frac{4 n-3}{4} \pi}$ with $n=1,2$, for $N=3, \theta_{n}=e^{j\left(\frac{1}{9}+\frac{2(n-1)}{3}\right) \pi}$ with $n=1,2,3$, and for $N=4, \theta_{n}=e^{j \frac{4 n-3}{8} \pi}$ with $n=1,2,3,4$. In the simulations, the user nodes have the same distance from $D$, denoted by $d$, and the channel variance between the user nodes to $D$ is assumed to be 1 . The inter-user distances are the same and denoted by $d_{u}$ with $d_{u}<d$. The path loss exponent is $\nu=3$. The total transmit power of each user for one transmission is $P_{t}=P_{I}+P_{I I}$, and $P_{I}=\alpha P_{t}$. In this work, we do not derive the optimal value for $\alpha$ because solving the optimization problem requires CSI of all the links known at each user node or a central controller. Such an optimization problem is out of the scope of this paper. However, the influence of $\alpha$ on the performance of the proposed schemes is given in the simulations. For the narrow-band cooperative communications, the channel coefficients follow the Jakes' model [33] with Doppler frequency $f_{D}$ and normalized fading parameter $f_{D} T_{s}$, where $T_{s}$ is the sampling period which equals the symbol period. Unless specified otherwise, $f_{d} T_{s}=0.0025$ is used throughout the simulation. 


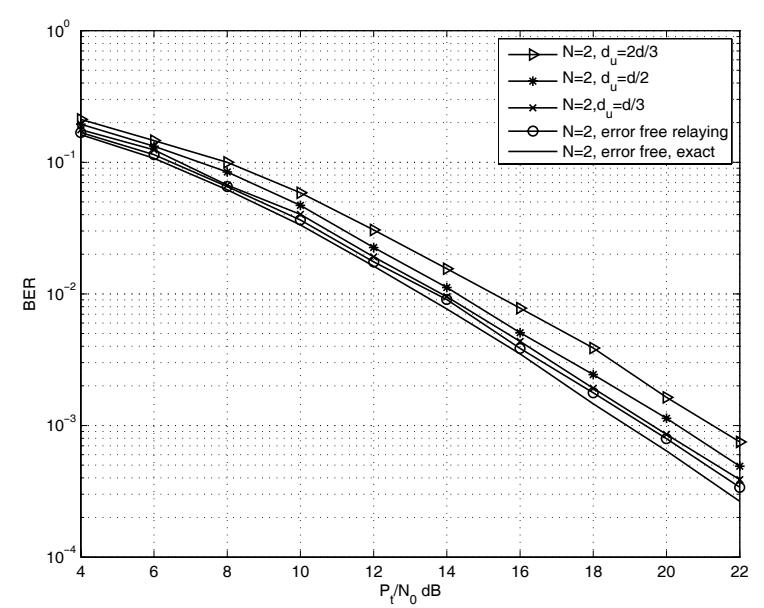

Fig. 6. BER performance of DSTNC for different distance between the user nodes with $N=2$.

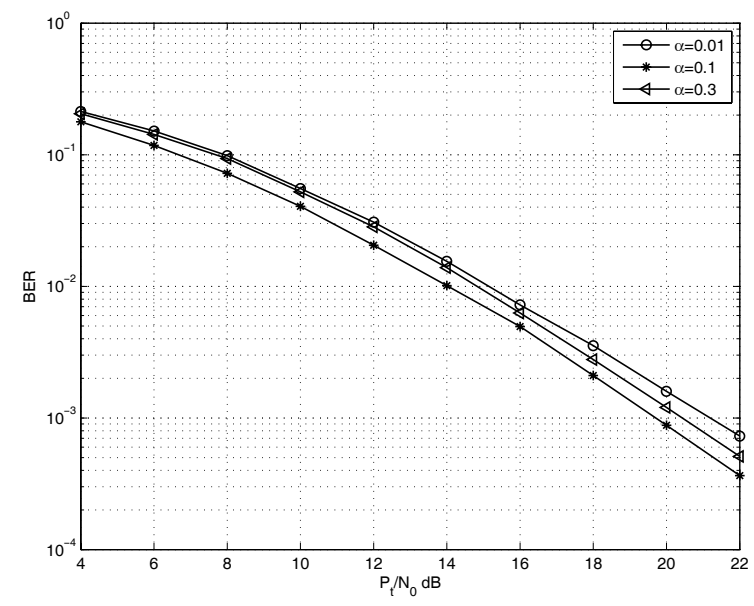

Fig. 7. The influence of different $\alpha$ on BER performance of DSTNC with $N=2$.

Fig. 5 shows the BER performance versus SNR $P_{t} / N_{0}$ of the DSTNC scheme with $N=2,3,4$ user nodes. In the simulation, $\alpha=0.1, d_{u}=d / 3$ and BPSK is adopted. From the dashed and solid curves, we can see that the BER performances for a certain number of user nodes are the same no matter the detection states of the user nodes are known at $D$ or not. From the curves, we verify that the full diversity order, which is equal to the number of user nodes $N$, is achieved by the proposed DSTNC scheme. The single node scenario without cooperation is also considered for a comparison. The user node without cooperation transmits the same symbol with the same power $P_{t}$. It can be seen clearly from Fig. 5 that the BER performance of single node without cooperation is improved by user cooperation, and the improvement becomes more significant as the number of cooperation nodes increases, due to the increase in diversity order. When SNR is low, the cooperative transmissions show no performance advantage. This is because the user nodes do not relay most of the received symbols due to the high BER. To compare with the same transmit bit rate, we also

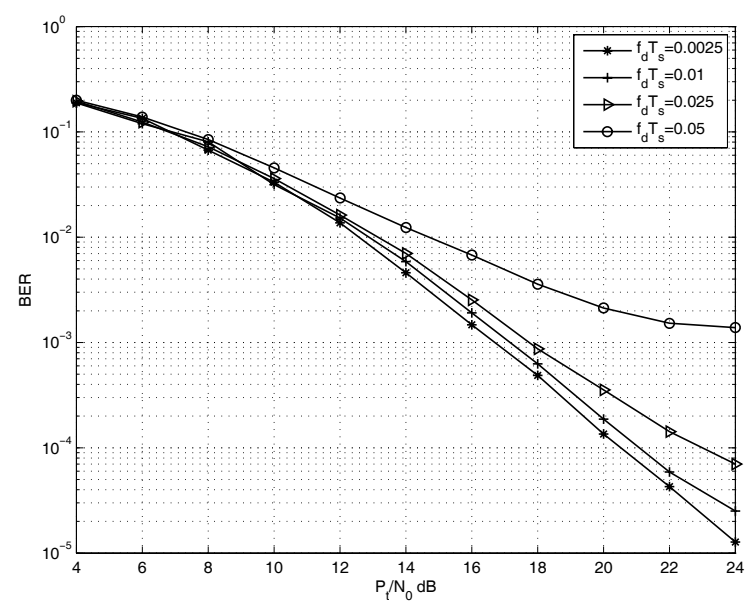

Fig. 8. BER performance of DSTNC for different Doppler frequency with $N=4$.

give the BER curve for $N=2$ users with QPSK. We can see that the BER performance with QPSK is worse than that with BPSK due to the smaller coding advantage of QPSK. But the cooperative transmission with QPSK outperforms the noncooperative transmission when SNR is high enough because of higher diversity order. For BER of practical interest, which is usually better than $10^{-3}$, cooperative transmission is better than non-cooperative transmission with the same transmission rate.

Fig. 6 depicts the BER performances for different interuser distances with $N=2$ and $\alpha=0.1$. It can be seen from Fig. 6 that, as the inter-user distance $d_{u}$ decreases, the BER performance becomes better. When the inter-user distance is one third of the distance between the user nodes and the destination, the performance of the proposed scheme with BPSK modulation is almost the same as that of the error-free relaying case. This performance is similar to that of a MISO system with $N$ transmit antennas. Compared to the error-free relaying case, the performance degradation increases as $d_{u}$ comes close to $d$. These observations match the analysis in Section III. From Theorem 1 we can see that the PEP is influenced by the inter-user distance through $\sigma_{l n}^{2} \propto d_{l n}^{-\alpha}$. Therefore, when the nodes are closer to each other, the channel variance becomes larger and better performance can be achieved. In many practical situations with adjacent cooperative nodes, the proposed DSTNC scheme can obtain a performance similar to the MISO system while overcoming some practical issues in cooperative communications. In the differential detection of (9) and (11), the signal terms in $\varepsilon_{m}$ and $\xi_{m}$ are replaced by their mean values 1 . To show the effect of this substitution, the BER performance of the ideal case which is error-free relaying and uses exact values of the symbols in detection is also plotted in Fig. 6. From the solid curve and the curve with circles we can see that, compared to the ideal case, the BER performance with mean value is slightly worse.

Fig. 7 shows the influence of $\alpha$ on the BER performance of the proposed DSTNC scheme. In the simulation, $N=2$ users. BPSK is used for the proposed DSTNC scheme. First 
TABLE I

Nokia Rooftop Channel 10-RAY POWER Delay Profile

\begin{tabular}{|c|c|c|c|c|c|c|c|c|c|c|}
\hline \hline Delay profile $(\mu s)$ & 0 & 0.03 & 0.07 & 0.11 & 0.15 & 0.19 & 0.23 & 0.27 & 0.31 & 0.35 \\
\hline Power Profile & 0.7094 & 0.1782 & 0.0577 & 0.0310 & 0.0126 & 0.0060 & 0.0028 & 0.0011 & 0.0009 & 0.0004 \\
\hline \hline
\end{tabular}

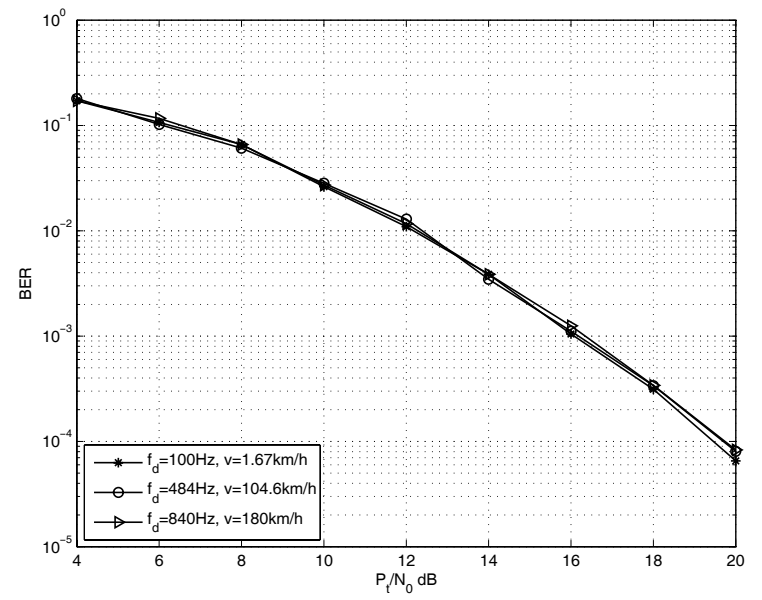

Fig. 9. BER performance of DSTFNC for different Doppler frequency with $N=2$.

we can see that different value of $\alpha$ does not affect the slope of the performance curve but only shifts it. In order words, the diversity order is invariant with $\alpha$ while the coding advantage depends on it, as discussed in Section III.A. Since the total power $P_{t}$ is fixed, larger $\alpha$ can get better BER performance at the user nodes, but degrade the relaying performance in Phase II. Smaller $\alpha$ means lager transmit power for Phase II, but the BER in Phase I becomes higher, which may influence the overall performance.

Fig. 8 presents the BER performance of the DSTNC scheme for different Doppler frequencies. Assume that $N=4$, $d_{u}=d / 3, \alpha=0.1$ and BPSK modulation is used. Because the differential scheme requires the channels do not change significantly during a period of two adjacent symbols, the performance degrades as the Doppler frequency increases, associated with decreasing channel coherence time. We can see from Fig. 8 that the performance degradation is quite small when $f_{d} T_{s}$ increases to 0.01 , but error floors appear when $f_{d} T_{s} \geq 0.025$. Although the performance of DSTNC degrades when the channel changes rapidly, it still can work in some situations where coherent detection is impractical. For example, considering a vehicle transmitting at a symbol rate of $30 \mathrm{kHz}$ and a frequency of $1.9 \mathrm{GHz}$. When $f_{d} T_{s}=0.025$, the vehicle moves at about $426 \mathrm{~km} / \mathrm{h}$, which is approaching the required speed for trains in third-generation European cellular standards [23], and the coherence time is on the order of 20 symbols. If five training symbols were used per antenna pair [23], a cooperative communication system with $N=4$ users would require about 20 training symbols for the channel estimations, which is a $100 \%$ overhead.

The BER performances of the DSTFNC scheme for broadband cooperative communications are given in Fig. 9 and Fig. 10. The DSTFNC is mapped from the DSTNC used in

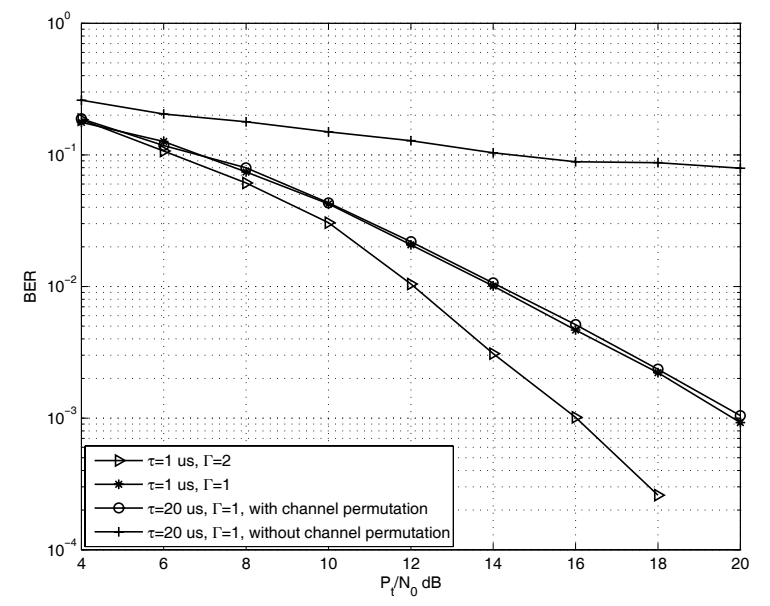

Fig. 10. BER performance of DSTFNC with different achievable diversity for different $\Gamma$ and channel permutation, $N=2$.

the previous simulations. There are $N=2$ user nodes in the cooperative system and $\alpha=0.1$.

The influence of Doppler effects is investigated in Fig. 9. In this simulation, non-line of sight (NLOS) Nokia rooftop wideband channel model in a suburban environment [34] is used. Table I shows a 10-tap delay line model to model its average power-delay profile. Each OFDM modulator has $N_{c}=64$ subcarriers with the total bandwidth of $20 \mathrm{MHz}$ and works in $5 \mathrm{GHz}$ band. The OFDM symbol duration is $3.2 \mu \mathrm{s}$ and the length of cyclic prefix is $0.8 \mu \mathrm{s}$. Different Doppler effects caused by different speeds are considered in Fig. 9. For walking speed $v=1.67 \mathrm{~km} / \mathrm{h}(6 \mathrm{~m} / \mathrm{s})$, the Doppler frequency offset $f_{d}$ is so small that the channel can be regarded as unchanged in one OFDM block. As speed increases, Doppler effect becomes more severe. However, as can be seen from Fig. 9, the BER performances of the DSTFNC scheme with $v=104.6 \mathrm{~km} / \mathrm{h}$ and $180 \mathrm{~km} / \mathrm{h}$ are similar to that with $v=1.67 \mathrm{~km} / \mathrm{h}$. That means the proposed differential scheme can work in some high mobility scenarios.

In Fig. 10, we consider a two-ray equal power delay profile with a delay of $\tau \mu s$ between the two rays to show the effects of diversity and channel permutation. Each OFDM modulator has $N_{c}=128$ subcarriers with the total bandwidth of $1 \mathrm{MHz}$. The corresponding OFDM block interval is $T=128 \mu \mathrm{s}$ without the cyclic prefix. In this simulation, the fading channels are assumed to be constant within each OFDM block and changes independently from one OFDM block to another. $\tau=1 \mu \mathrm{s}$ and $20 \mu \mathrm{s}$ are considered. Since the separation of $\tau=1 \mu \mathrm{s}$ is very small compared with $T=128 \mu \mathrm{s}$, the channel frequency responses change smoothly over different subcarriers, and the differential encoding is performed over adjacent subcarriers. We can see that the DSTFNC scheme performs successfully in this case. The effect of different $\Gamma$ is investigated in 


$$
P\left(\mathbf{s}^{k} \rightarrow \mathbf{c}^{k}\right) \leq \prod_{n=1}^{N}\left[\frac{4 \varepsilon_{n}}{(1-\alpha) \sigma_{n}^{2}\left|\mathbf{a}_{n}^{\mathrm{T}} \Delta \mathbf{s}\right|^{2}}\left(\frac{P_{t}}{N_{0}}\right)^{-1}-\sum_{m=1, m \neq n}^{N} \frac{C_{M} \xi_{m}}{\alpha \sigma_{m n}^{2}} \frac{4 \varepsilon_{n}}{(1-\alpha) \sigma_{n}^{2}\left|\mathbf{a}_{n}^{\mathrm{T}} \Delta \mathbf{s}\right|^{2}}\left(\frac{P_{t}}{N_{0}}\right)^{-2}+\sum_{l=1, l \neq n}^{N} \frac{C_{M} \xi_{l}}{\alpha \sigma_{l n}^{2}}\left(\frac{P_{t}}{N_{0}}\right)^{-1}+\text { H.O.T. }\right]
$$

$$
P\left(\mathbf{s}^{k} \rightarrow \mathbf{c}^{k}\right) \leq \prod_{n=1}^{N}\left[\left(\frac{P_{t}}{N_{0}}\right)^{-1} \frac{1}{\left|\mathbf{a}_{n}^{\mathrm{T}} \Delta \mathbf{s}\right|^{2}}\left(\frac{4 \varepsilon_{n}}{(1-\alpha) \sigma_{n}^{2}}+\sum_{l=1, l \neq n}^{N} \frac{C_{M} \xi_{l}\left|\mathbf{a}_{n}^{\mathrm{T}} \Delta \mathbf{s}\right|^{2}}{\alpha \sigma_{l n}^{2}}\right)\right] .
$$

Fig. 10. To compare with the same bit rate, QPSK is used for $\Gamma=2$ and BPSK is used for $\Gamma=1$. It is obvious that the BER performance with $\Gamma=2$ is much better than that with $\Gamma=1$ because higher diversity is achieved when $\Gamma=2$, which exploits both spatial and frequency diversity. In case of $\tau=20 \mu \mathrm{s}$, the channel frequency responses change severely. Therefore, as shown in Fig. 10, the DSTFNC scheme which differentially encoded over adjacent subcarriers fails to work. However, by utilizing the knowledge of statistical channel PDP, each user node can permutate the channel and obtain a smooth logical channel to guarantee the successful differential decoding. As shown by the curves with crosses and stars in Fig. 10, when $\Gamma=1$, by channel permutation, the BER performance of $\tau=20 \mu \mathrm{s}$ is almost the same as that of $\tau=1 \mu s$.

\section{CONCLUSION}

In this paper, we consider the practical challenges in timing and frequency synchronization and channel estimation for multi-source cooperative communications and propose new cooperative differential transmission schemes for narrowband and broadband systems, called distributed DSTNC and DSTFNC, respectively, to overcome such issues. Compared with the traditional multi-source cooperative communication using TDMA, the proposed DSTNC can achieve full diversity with a significant reduction in transmission delay. The proposed schemes utilize linear network coding to combine the information and reduce the required time slot. The PEP performance of DSTNC is analyzed, and design criteria of the network coding vectors are derived. The performance with small channel coherence time is also studied to investigate the feasibility of DSTNC. The distributed DSTFNC is designed through mapping from DSTNC. The differential encoding is performed in frequency domain within each OFDM block. Despite the Doppler effects caused by mobility, the DSTFNC can still be decoded in high mobility scenarios. When the statistical channel PDP is known at the corresponding user node, the performance of the DSTFNC is improved by independent channel permutation at each user node. Simulation results are shown to validate our analysis.

\section{APPENDIX}

This appendix presents the proof of Theorem 1.

The SER for M-QAM modulation can be expressed as [35] $p_{m n}=F_{2}\left(1+\frac{b_{q} \gamma_{m n}}{\sin ^{2} \theta}\right)$, where $b_{q}=\frac{b_{Q A M}}{2}=\frac{3}{2(M-1)}$ and $\gamma_{m n}$ is the average received SNR at $U_{n}$ for the symbols from $U_{m} \cdot \gamma_{m n}$ can be given as $\gamma_{m n}=\frac{\sigma_{m n}^{2} P_{I}}{\xi_{m} N_{0}}$ where $\xi_{m}$ has been defined in Section II. B. The function $F_{2}(x(\theta))$ is given as [35]

$$
F_{2}(x(\theta))=\frac{4 K}{\pi} \int_{0}^{\frac{\pi}{2}} \frac{1}{x(\theta)} d \theta-\frac{4 K^{2}}{\pi} \int_{0}^{\frac{\pi}{4}} \frac{1}{x(\theta)} d \theta
$$

with $K=1-\frac{1}{\sqrt{M}}$. Let $C_{M}$ equal the following constant

$$
C_{M}=\frac{4 K}{\pi b_{q}} \int_{0}^{\frac{\pi}{2}} \sin ^{2} \theta d \theta-\frac{4 K^{2}}{\pi b_{q}} \int_{0}^{\frac{\pi}{4}} \sin ^{2} \theta d \theta .
$$

When SNR is high, $1+\frac{b_{q} \gamma_{m n}}{\sin ^{2} \theta} \approx \frac{b_{q} \gamma_{m n}}{\sin ^{2} \theta}$ and $p_{m n}$ can be approximated as

$$
p_{m n}=C_{M} \gamma_{m n}^{-1} .
$$

Let $\alpha=\frac{P_{I}}{P_{t}}$. Substituting (42) into (22) and ignoring the 1 in the denominator for high SNR, we can get (43) on the top of the this page. For high SNR, the term of $\left(\frac{P_{t}}{N_{0}}\right)^{-2}$ and H.O.T can be ignored. Then (43) becomes (44), which is shown on the top of the this page.

Because $\mathbf{a}_{n}^{\mathrm{T}} \mathbf{a}_{n}=1$, the maximum value of $\left|\mathbf{a}_{n}^{\mathrm{T}} \Delta \mathbf{s}\right|$ is $\sqrt{N} d_{\max }$, where $d_{\max }$ denotes the maximum Euclidean distance of $\mathcal{A}$. For example, if $\mathcal{A}$ is a 4-QAM constellation, the maximum can be achieved when $\Delta s_{m}=\sqrt{2}(1+j)$, and $a_{m n}=\frac{1}{\sqrt{N}} e^{-j \frac{\pi}{4}}, m \in[1, N]$. Therefore, (44) can be upper bounded by

$P\left(\mathbf{s}^{k} \rightarrow \mathbf{c}^{k}\right)<\prod_{n=1}^{N}\left[\left(\frac{P_{t}}{N_{0}}\right)^{-1} \frac{1}{\left|\mathbf{a}_{n}^{\mathrm{T}} \Delta \mathbf{s}\right|^{2}}\left(\frac{4 \varepsilon_{n}}{\sigma_{n}^{2}}+\sum_{l=1, l \neq n}^{N} \frac{C_{M} \xi_{l} N d_{\max }^{2}}{\sigma_{l n}^{2}}\right)\right]$.

The upper bound in Theorem 1 is obtained.

\section{REFERENCES}

[1] J. N. Laneman, D. N. C. Tse, and G. W. Wornell, "Cooperative diversity in wireless networks: efficient protocols and outage behavior," IEEE Trans. Inf. Theory, vol. 50, no. 12, pp. 3062-3080, Dec. 2004.

[2] V. Tarokh, N. Seshadri, and A. R. Calderbank, "Space-time codes for high data rate wireless communication: performance criterion and code construction," IEEE Trans. Inf. Theory, vol. 44, no. 2, pp. 744-765, Mar. 1998.

[3] W. Su, X. G. Xia, and K. J. R. Liu, "A systematic design of highrate complex orthogonal space-time block codes," IEEE Commun. Lett., vol. 8, no. 6, pp. 380-382, 2004.

[4] J. N. Laneman and G. W. Wornell, "Distributed space-time-coded protocols for exploiting cooperative diversity in wireless network," IEEE Trans. Inf. Theory, vol. 49, no. 10, pp. 2415-2425, Oct. 2003.

[5] A. K. Sadek, W. Su, and K. J. R. Liu, "Multinode cooperative communications in wireless networks," IEEE Trans. Signal Process., vol. 55, no. 1, pp. 341-355, Jan. 2007.

[6] Q. Zhao and H. Li, "Differential modulation for cooperative wireless systems," IEEE Trans. Signal Process., vol. 55, no. 5, pp. 2273-2283, May 2007.

[7] Y. Shang and X. G. Xia, "Shift-full-rank matrices and applications in space-time trellis codes for relay networks with asynchronous cooperative diversity," IEEE Trans. Inf. Theory, vol. 52, no. 7, pp. 3153-3167, July 2006. 
[8] M. O. Damen and A. R. Hammons, "Delay-tolerant distributed TAST codes for cooperative diversity," IEEE Trans. Inf. Theory, vol. 53, no. 10, pp. 3755-3773, Oct. 2007.

[9] S. Wei, D. Goeckel, and M. Valenti, "Asynchronous cooperative diversity," IEEE Trans. Wireless Commun., vol. 5, no. 6, pp. 1547-1557, June 2006.

[10] M. Sharp, A. Scaglione, and B. Sirkeci-Mergen, "Randomized cooperation in asynchronous dispersive links," IEEE Trans. Commun., vol. 57, no. 1, pp. 64-68, Jan. 2009.

[11] X. Li, "Space-time coded multi-transmission among distributed transmitters without perfect synchronization," IEEE Signal Process. Lett., vol. 11, no. 12, pp. 948-951, Dec. 2004.

[12] A. Ribeiro, R. Wang, and G. B. Giannakis, "Multi-source cooperation with full-diverisy spectral-efficiency and controllable-complexity," IEEE J. Sel. Areas Commun., vol. 25, no. 2, pp. 415-425, Feb. 2007.

[13] A. Cano, J. Gomez-Vilardebo, A. I. Perez-Neira, and G. B. Giannakis, "High-rate distributed multi-source cooperation using complex field coding," in Proc. ICASSP, Apr. 2009, pp. 2633-2636.

[14] H. Q. Lai, A. Ibrahim, and K. J. R. Liu, "Wireless network cocast: Location-aware cooperative communications with linear network coding," IEEE Trans. Wireless Commun., vol. 8, no. 6, pp. 1-11, June 2009.

[15] H. Q. Lai and K. J. R. Liu, "Space-time network coding," IEEE Trans. Signal Process., vol. 59, no. 4, pp. 1706-1718, Apr. 2011.

[16] Y. Jing and H. Jafarkhani, "Distributed differential space-time coding for wireless relay networks," IEEE Trans. Commun., vol. 56, no. 7, pp. 1092-1100, July 2008.

[17] T. Himsoon, W. P. Siriwongpairat, W. Su, and K. J. R. Liu, "Differential modulation for multinode cooperative communications," IEEE Trans. Signal Process., vol. 56, no. 7, pp. 2941-2956, July 2008.

[18] S. Y. R. Li, W. Yeung, and N. Cai, "Linear network coding," IEEE Trans. Inf. Theory, vol. 49, no. 2, pp. 371-381, Feb. 2003.

[19] E. Fasolo, F. Rossetto, and M. Zorzi, "Network coding meets MIMO," in Proc. Workshop Network Coding, Theory, Appl., Jan. 2008, pp. 1-6.

[20] W. Su and K. J. R. Liu, "Differential space-frequency modulation via smooth logical channel for broadband wireless communications," IEEE Trans. Commun., vol. 53, no. 12, pp. 2024-2028, Dec. 2005.

[21] M. Damen, A. Chkeif, and J. Belfiore, "Lattice code decoder for spacetime codes," IEEE Signal Process. Lett., vol. 4, no. 5, pp. 161-163, May 2000.

[22] Z. Safar, W. Su, and K. J. R. liu, "A fast sphere decoding framework for space-frequency block codes," in Proc. ICC, 2004, pp. 2591-2595.

[23] B. L. Hughes, "Differential space-time modulation," IEEE Trans. Inf. Theory, vol. 46, no. 7, pp. 2567-2578, Nov. 2000.

[24] B. M. Hochwald and T. L. Marzetta, "Unitary space-time modulation for multiple-antenna communications in Rayleigh flat fading," IEEE Trans. Inf. Theory, vol. 46, no. 2, pp. 543-564, Mar. 2000.

[25] V. Tarokh and H. Jafarkhani, "A differential detection scheme for transmit diversity," IEEE J. Sel. Areas Commun., vol. 18, no. 7, pp. $1169-1174$, July 2000.

[26] V. Tarokh, H. Jafarkhani, and A. R. Calderbank, "Spcae-time block codes from orthogonal design," IEEE Trans. Inf. Theory, vol. 45, no. 5, pp. 1456-1467, July 1999.

[27] X. Giraud, E. Boutillon, and J. C. Belfiore, "Algebraic tools to build modulation schemes for fading channels," IEEE Trans. Inf. Theory, vol. 43, no. 3, pp. 938-952, May 1997.

[28] J. Boutros and E. Viterbo, "Signal space diversity: a power and bandwith efficient diversity technique for the Rayleigh fading channel," IEEE Trans. Inf. Theory, vol. 44, no. 4, pp. 1453-1467, July 1998.

[29] W. Su, Z. Safar, and K. J. R. Liu, "Signal space diversity: a power and bandwith efficient diversity technique for the Rayleigh fading channel," IEEE Trans. Wireless Commun., vol. 4, no. 4, pp. 1847-1857, July 2005.

[30] — - "Full-rate full-diversity space-frequency codes with optimum coding advantage," IEEE Trans. Inf. Theory, vol. 51, no. 1, pp. 229-249, Jan. 2005.
[31] W. Su, Z. Safar, M. Olfat, and K. J. R. Liu, "Obtain full-diversity spacefrequency codes from space-time codes via mapping," IEEE Trans. Signal Process., vol. 51, no. 11, pp. 2905-2916, Nov. 2003.

[32] G. Gallo and S. Pallotino, "Shortest path methods: a unifying approach," Math. Program. Study, vol. 26, pp. 2905-2916, Nov. 1986.

[33] W. C. Jakes, Microwave Mobile Communicationss. Wiley-IEEE Press, 1994.

[34] Y. Chen, E. Aktas, and U. Tureli, "Optimal space-frequency group codes for MIMO-OFDM system," IEEE Trans. Commun., vol. 54, no. 3, pp. 553-562, Mar. 2006.

[35] K. J. R. Liu, A. K. Sadek, W. Su, and A. Kwasinski, Cooperative Communications and Networking. Cambridge University Press, 2008.

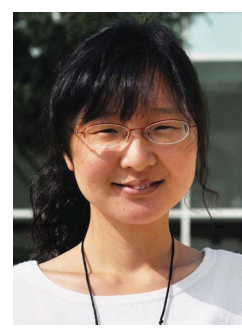

Zhenzhen Gao (S'10) received the B.S. in communication engineering in 2005 from Lanzhou University, Lanzhou, China. She is now a Ph.D. student in the Department of Information and Communication Engineering, Xi' an Jiaotong University, Xi'an, China. She received a scholarship from the China Scholarship Council (CSC) in 2009. From September 2009 to August 2011, she was a visiting student in the Department of Electrical and Computer Engineering at the University of Maryland, College Park, MD, USA. Her current research interests are in the areas of wireless communications and networks, including cooperative communications, space-time coding, and network coding.

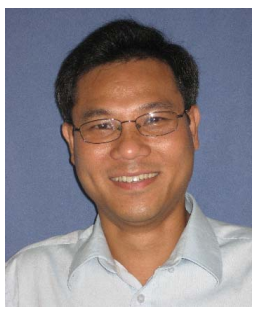

Hung-Quoc Lai (M'11) received the B.S. (cum laude) degree, the M.S. degree, and the Ph.D. degrees all in electrical engineering from the University of Maryland, College Park, Maryland in 2004, 2006, and 2011, respectively. Since 2006, he has been with the U.S. Army RDECOM CERDEC at Aberdeen Proving Ground, Maryland, where he currently is the Technical Lead in research areas of multiple-input multiple-output (MIMO) and cooperative communications. Dr. Lai is a Gates Millennium Scholar. $\mathrm{He}$ is the recipient of a university-level Distinguished Teaching Assistant Award from the University of Maryland and of the 2004-2005 George Corcoran Memorial Award, a lifetime award for his contributions to ECE education, the Department of Electrical and Computer Engineering, University of Maryland.

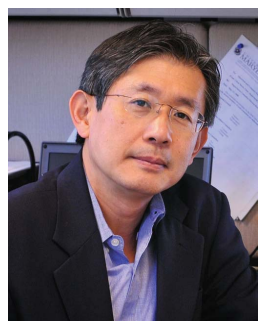

K. J. Ray Liu (F'03) became a Distinguished Scholar-Teacher of the University of Maryland, College Park, in 2007, where he is the Christine Kim Eminent Professor of Information Technology. He serves as Associate Chair of Graduate Studies and Research of the Electrical and Computer Engineering Department and leads the Maryland Signals and Information Group conducting research encompassing broad aspects of wireless communications and networking, information forensics and security, multimedia signal processing, and biomedical engi-

neering.

Dr. Liu is the recipient of numerous honors and awards including the IEEE Signal Processing Society Technical Achievement Award and is a Distinguished Lecturer. He also received various teaching and research recognitions from the University of Maryland including the university-level Invention of the Year Award; and the Poole and Kent Senior Faculty Teaching Award and Outstanding Faculty Research Award, both from the A. James Clark School of Engineering. An ISI Highly Cited Author in Computer Science, Dr. Liu is a Fellow of IEEE and AAAS.

Dr. Liu is President-Elect and was Vice President - Publications of the IEEE Signal Processing Society. He was the Editor-in-Chief of IEEE Signal Processing Magazine and the the founding Editor-in-Chief of the EURASIP Journal on Advances in Signal Processing. 